\title{
THE KINEMATICS OF THE ULTRA-FAINT MILKY WAY SATELLITES: SOLVING THE MISSING SATELLITE PROBLEM
}

\author{
JoshuA D. SIMON \\ Department of Astronomy, California Institute of Technology, 1200 East California Boulevard, \\ MS 105-24, Pasadena, CA 91125; jsimon@astro.caltech.edu \\ AND \\ Marla GeHA ${ }^{1}$ \\ National Research Council of Canada, Herzberg Institute of Astrophysics, 5071 West Saanich Road, \\ Victoria, BC V9E 2E7, Canada; marla.geha@nrc-cnrc.gc.ca \\ Received 2007 May 18; accepted 2007 July 21
}

\begin{abstract}
We present Keck DEIMOS spectroscopy of stars in eight of the newly discovered ultra-faint dwarf galaxies around the Milky Way. We measure the velocity dispersions of Canes Venatici I, Canes Venatici II, Coma Berenices, Hercules, Leo IV, Leo T, Ursa Major I, and Ursa Major II from the velocities of 18-214 stars in each galaxy and find dispersions ranging from 3.3 to $7.6 \mathrm{~km} \mathrm{~s}^{-1}$. The six galaxies with absolute magnitudes $M_{V}<-4$ are highly dark matter dominated, with mass-to-light ratios approaching $1000 M_{\odot} / L_{\odot, V}$. For the fainter galaxies we find tentative evidence for tidal disruption. The measured velocity dispersions of the ultra-faint dwarfs are correlated with their luminosities, indicating that a minimum mass for luminous galactic systems may not yet have been reached. We also measure the metallicities of the observed stars and find that the new dwarfs have mean metallicities of $[\mathrm{Fe} / \mathrm{H}]=-2.0$ to -2.3 ; these galaxies represent some of the most metal-poor stellar systems known. The six brightest of the ultra-faint dwarfs extend the luminosity-metallicity relationship followed by more luminous dwarfs by a factor of $\sim 30$ in luminosity. We detect metallicity spreads of up to 0.5 dex in several objects, suggesting multiple star formation epochs. UMa II and Com, despite their exceptionally low luminosities, have higher metallicities that suggest they may once have been much more massive. Having established the masses of the ultra-faint dwarfs, we re-examine the missing satellite problem. After correcting for the sky coverage of the Sloan Digital Sky Survey, we find that the ultra-faint dwarfs substantially alleviate the discrepancy between the predicted and observed numbers of satellites around the Milky Way, but there are still a factor of $\sim 4$ too few dwarf galaxies over a significant range of masses. We show that if galaxy formation in low-mass dark matter halos is strongly suppressed after reionization, the simulated circular velocity function of CDM subhalos can be brought into approximate agreement with the observed circular velocity function of Milky Way satellite galaxies.
\end{abstract}

Subject headings: dark matter — galaxies: dwarf — galaxies: kinematics and dynamics — Local Group techniques: radial velocities

Online material: color figure

\section{INTRODUCTION}

The cold dark matter (CDM) cosmological model predicts that massive galaxies such as the Milky Way should be surrounded by large numbers of dark matter dominated satellite halos. The relatively modest populations of observed dwarf galaxies orbiting the Milky Way and Andromeda, however, seem to conflict with this prediction (Kauffman et al. 1993; Klypin et al. 1999; Moore et al. 1999). This apparent disagreement between the expected and observed numbers of dwarf galaxies has become widely known as the "substructure" or "missing dwarf" problem.

Proposed solutions to the substructure problem can be broadly divided into two categories: cosmological and astrophysical. Cosmological solutions include modifying the power spectrum at small scales (Kamionkowski \& Liddle 2000; Zentner \& Bullock 2003) and changing the properties of the dark matter particles, such as by making them warm (Colín et al. 2000; Bode et al. 2001) or invoking a late decay from a nonrelativistic particle (Strigari et al. 2007b). Astrophysical solutions are more prosaic, but perhaps easier to constrain observationally. Some of the most popular astro-

\footnotetext{
${ }^{1}$ Current address: Astronomy Department, Yale University, New Haven, CT 06520; marla.geha@yale.edu.
}

physical solutions include the hypothesis that reionization could suppress the formation of dwarf galaxies by preventing low-mass dark matter halos from acquiring enough gas to form stars after $z \sim 10$ (e.g., Bullock et al. 2000; Somerville 2002; Benson et al. 2002; Ricotti \& Gnedin 2005; Moore et al. 2006) and the possibility that the dwarf galaxies we observe today were once much more massive objects that have been reduced to their present appearance by dramatic tidal stripping (Mayer et al. 2001a, 2001b; Kravtsov et al. 2004). Despite a wealth of ideas about how to solve the missing dwarf problem, distinguishing between the various proposals has proved to be difficult, and making sense of the tremendous variety of masses, luminosities, mass-to-light ratios, gas fractions, and star formation histories among observed dwarf galaxies remains a challenge.

Our understanding of the missing satellite problem and the evolution of dwarf galaxies is being rapidly revised by the discovery of a large population of new, very faint Local Group dwarfs in the Sloan Digital Sky Survey (SDSS; York et al. 2000) and other wide-field imaging surveys. In the past three years, at least 20 of these galaxies have been identified, nearly doubling the previously known population. The new dwarfs include eight additional Milky Way dwarf spheroidals (Willman et al. 2005a; Zucker et al. 2006a, 2006b; Belokurov et al. 2006, 2007b; Grillmair 2006; 
TABLE 1

OBSERVING TARGETS

\begin{tabular}{|c|c|c|c|c|c|c|}
\hline Galaxy & $\alpha(\mathrm{J} 2000.0)$ & $\delta(\mathrm{J} 2000.0)$ & $M_{V}$ & $\begin{array}{c}\mu_{V}^{\mathrm{a}} \\
\left(\mathrm{mag} \operatorname{arcsec}^{-2}\right)\end{array}$ & $\begin{array}{l}\text { Distance }^{\mathrm{b}} \\
\quad(\mathrm{kpc})\end{array}$ & References \\
\hline Ursa Major II ...................... & 085130.00 & 630748.0 & -3.8 & 28.8 & 32 & 1,2 \\
\hline Leo T................................... & 093453.40 & 170305.0 & -7.1 & 26.9 & 417 & 3 \\
\hline 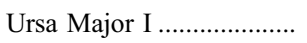 & 103452.80 & 515512.0 & -5.6 & 28.9 & 106 & $4,5,6$ \\
\hline 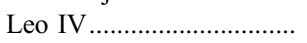 & 113257.00 & -003200.0 & -5.1 & 28.3 & 158 & 2 \\
\hline Coma Berenices .................. & 122659.00 & 235415.0 & -3.7 & 27.4 & 44 & 2 \\
\hline Canes Venatici II................. & 125710.00 & 341915.0 & -4.8 & 27.2 & 151 & 2 \\
\hline Canes Venatici I.................... & 132803.50 & 333321.0 & -7.9 & 28.2 & 224 & 7 \\
\hline 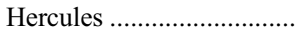 & 163102.00 & 124730.0 & -6.0 & 28.6 & 138 & 2 \\
\hline
\end{tabular}

NoтE.-Units of right ascension are hours, minutes, and seconds, and units of declination are degrees, arcminutes, and arcseconds.

${ }^{a}$ Central surface brightnesses, calculated from the Plummer profile fit parameters given in the cited discovery papers.

b The distances reported in the literature for these galaxies have generally been rounded off to the nearest multiple of $10 \mathrm{kpc}$ after converting from the distance modulus, which is the quantity directly constrained by the data. The distances listed here have been calculated from the published distance moduli and rounded to the nearest kpc.

REFERENCES.- (1) Zucker et al. 2006b; (2) Belokurov et al. 2007b; (3) Irwin et al. 2007; (4) Willman et al. 2005a; (5) Belokurov et al. 2006; (6) this work; (7) Zucker et al. 2006a.

Sakamoto \& Hasegawa 2006) and one dwarf irregular (Irwin et al. 2007), eight new dwarf spheroidals around Andromeda (Zucker et al. 2004, 2007; Martin et al. 2006; Majewski et al. 2007; Ibata et al. 2007), and three further new Milky Way satellites that lie in the uncertain parameter space between dwarf galaxies and globular clusters (Willman et al. 2005b; Belokurov et al. 2007b; Walsh et al. 2007). Nearly all of these objects have both surface brightnesses and luminosities that are significantly lower than those of any previously known galaxies.

Properly placing these new discoveries within the framework of CDM and the missing satellite problem requires measurements of their internal kinematics, in order to determine whether the ultrafaint dwarfs are gravitationally bound, dark matter dominated galaxies, or tidally disrupted systems. Only five of these objects (Ursa Major I, Andromeda IX, Boötes, Canes Venatici I, and Andromeda XIV) have published stellar kinematics measurements, and for two of the three ultra-faint Milky Way dwarfs that have been studied already only a handful of stars were observed (Kleyna et al. 2005; Chapman et al. 2005; Muñoz et al. 2006a; Ibata et al. 2006; Majewski et al. 2007). In this paper, we present new stellar velocity measurements of larger samples of stars in eight of the 12 new Milky Way satellites (see Table 1). Including the other published studies and studies in preparation that we are aware of, the only known Milky Way satellites that remain unobserved are Segue 1 and Boötes II.

In $\S 2$, we describe our observations, target selection, and data reduction, focusing in particular on our techniques for obtaining very high precision velocity measurements with the Deep Imaging Multi-Object Spectrograph (DEIMOS). We present the main results of this study, including measured velocity dispersions, masses, mass-to-light ratios, and metallicities in $\S 3$. In $\S 4$, we discuss the implications of our results for the CDM model and the missing satellite problem. We summarize our results and conclusions in $\S 5$.

\section{OBSERVATIONS AND DATA REDUCTION}

\subsection{Observations}

We obtained spectra of individual stars in eight dwarf galaxies with the DEIMOS spectrograph (Faber et al. 2003) on the Keck II telescope on 2007 February 12-14. During the observations, the weather was clear, with seeing that varied between $0.5^{\prime \prime}$ and $0.9^{\prime \prime}$ (with a very brief excursion to $1.4^{\prime \prime}$ ). The spectrograph was configured to cover the wavelength range $6500-9000 \AA$ with the 1200 line $\mathrm{mm}^{-1}$ grating, and the OG550 filter was used to block shorter wavelength light. The spectral dispersion of this setup is $0.33 \AA$ pixel $^{-1}$, and the resulting spectral resolution, taking into account our slit width of $0.7^{\prime \prime}$ and the anamorphic distortion factor of 0.7 , is $1.37 \AA$ FWHM (corresponding to $12 \mathrm{~km} \mathrm{~s}^{-1}$ pixel $^{-1}$ and $47 \mathrm{~km} \mathrm{~s}^{-1} \mathrm{FWHM}$ at the Ca II triplet). Exposures of $\mathrm{Kr}, \mathrm{Ar}, \mathrm{Ne}$, and $\mathrm{Xe}$ arc lamps provided the wavelength calibration, and an internal quartz lamp was used for flat-fielding.

We observed 18 DEIMOS slit masks, with total exposure times ranging between 20 minutes and $2.5 \mathrm{hr}$. One to four masks were placed on each galaxy. Each mask contained 50-100 stars, of which $\sim 30 \%-80 \%$ were expected to be actual members of the target galaxies from the SDSS photometry. The positions, exposure times, and number of slits on each mask are listed in Table 2. Typical target stars had magnitudes of $r \approx 20-21$. At $r=20$, a $1 \mathrm{hr}$ exposure in good seeing conditions yields a signal-to-noise ratio $(\mathrm{S} / \mathrm{N})$ of $\sim 15$, and a $2.5 \mathrm{hr}$ exposure gives a $\mathrm{S} / \mathrm{N}$ of $\sim 22$, where the $\mathrm{S} / \mathrm{N}$ is calculated as the average $\mathrm{S} / \mathrm{N}$ per pixel in the $\mathrm{Ca}$ II triplet region.

Target selection was carried out on star catalogs extracted from the NYU-VAGC analysis (Blanton et al. 2005) of the Sloan Digital Sky Survey Data Release 5 data set (Adelman-McCarthy et al. 2007). ${ }^{2}$ We set the target priorities so as to preferentially observe stars with a high likelihood of being members of the various dwarfs based on their color, apparent magnitude, and position. We constructed $r, g-i$ color-magnitude diagrams (CMDs) for each dwarf and overlaid globular cluster isochrones from Clem (2005, hereafter $\mathrm{C} 05$ ), adjusted for the distance reported in the literature. We chose the best-fitting globular cluster red giant branch (RGB) of the three examples provided by $\mathrm{C} 05$. We also added a horizontal branch track derived from the M13 observations of C05 and asymptotic giant branch (AGB) isochrones (for an age of $11.2 \mathrm{Gyr}$ and a metallicity of $[\mathrm{Fe} / \mathrm{H}]=-1.3$ or $[\mathrm{Fe} / \mathrm{H}]=-1.7$ ) from Girardi et al. (2004). The highest priority targets were those located within 0.1 mag (in the least-squares sense ${ }^{3}$ ) of the RGB or AGB tracks, or within $0.2 \mathrm{mag}$ of the horizontal branch, with additional preference being given to brighter stars. Stars farther from any of the

\footnotetext{
2 The position of Leo T, which was discovered during our observing preparations, had not yet been processed for the VAGC at that time, so to select targets for that galaxy we used the standard DR5 data.

As in $\S 3.1$, when we refer to the distance between a star and a fiducial track in a color-magnitude diagram, we mean the following: $d_{\mathrm{CMD}}=\left\{\left[(g-i)_{*}-(g-\right.\right.$ $\left.\left.i)_{\text {fiducial }}\right]^{2}+\left(r_{*}-r_{\text {fiducial }}\right)^{2}\right\}^{1 / 2}$, where the appropriate reference point for each star along the fiducial track is chosen so as to minimize $d_{\mathrm{CMD}}$.
} 
TABLE 2

Keck Deimos Slit Mask Observing Parameters

\begin{tabular}{|c|c|c|c|c|c|c|}
\hline Mask Name & $\alpha(\mathrm{J} 2000.0)$ & $\delta(\mathrm{J} 2000.0)$ & $\begin{array}{l}\text { P.A. } \\
(\operatorname{deg})\end{array}$ & $\begin{array}{l}t_{\exp } \\
(\mathrm{s})\end{array}$ & $\begin{array}{c}\text { Number } \\
\text { of Slits }\end{array}$ & $\begin{array}{l}\text { Percent Useful } \\
\text { Spectra }\end{array}$ \\
\hline UMaII-1 ........................... & 085038.68 & 630645.0 & 95.8 & 3600 & 81 & $48 \%$ \\
\hline UMaII-2 …….................... & 084942.19 & 631105.6 & 180.0 & 3600 & 87 & $52 \%$ \\
\hline UMaII-3 ................................ & 085308.75 & 630445.4 & 109.0 & 2400 & 76 & $62 \%$ \\
\hline UMaI-1 ................................. & 103450.57 & 515447.7 & 65.0 & 5400 & 68 & $59 \%$ \\
\hline UMaI-2 ….............................. & 103422.23 & 515623.9 & 66.0 & 3600 & 62 & $65 \%$ \\
\hline 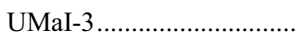 & 103535.62 & 515606.4 & 23.3 & 5400 & 68 & $85 \%$ \\
\hline 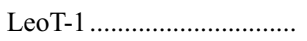 & 093500.18 & 170056.3 & 1.0 & 3600 & 87 & $75 \%$ \\
\hline 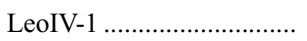 & 113258.69 & -003141.1 & 9.8 & 3000 & 77 & $83 \%$ \\
\hline 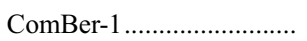 & 122708.32 & 235252.0 & 117.0 & 9000 & 78 & $62 \%$ \\
\hline ComBer-2 …........................... & 122644.48 & 235758.7 & 140.0 & 9000 & 78 & $51 \%$ \\
\hline 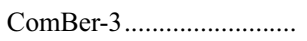 & 122647.98 & 235442.8 & -20.0 & 9000 & 80 & $65 \%$ \\
\hline 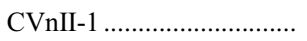 & 125712.78 & 342043.8 & -20.0 & 9000 & 67 & $81 \%$ \\
\hline 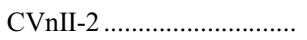 & 125716.03 & 341851.8 & 50.0 & 1200 & 66 & $30 \%$ \\
\hline 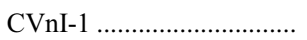 & 132759.38 & 333426.8 & 73.0 & 4140 & 91 & $87 \%$ \\
\hline 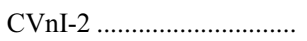 & 132809.19 & 333116.0 & 70.5 & 4140 & 94 & $83 \%$ \\
\hline 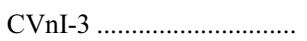 & 132814.34 & 333323.3 & -2.0 & 4860 & 90 & $83 \%$ \\
\hline 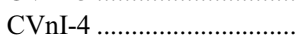 & 132802.17 & 333336.7 & -112.0 & 9000 & 115 & $79 \%$ \\
\hline 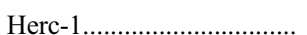 & 163102.70 & 124721.3 & 104.0 & 4500 & 106 & $83 \%$ \\
\hline
\end{tabular}

Noтеs.-Mask name, right ascension, declination, position angle, and total exposure time for each Keck DEIMOS slit mask. Units of right ascension are hours, minutes, and seconds, and units of declination are degrees, arcminutes, and arcseconds. The final two columns refer to the total number of slitlets on each mask and the percentage of those slitlets for which a redshift was measured.

fiducial sequences were classified as lower priority targets. We then designed slit masks so as to maximize the number of high-priority targets while still obtaining good spatial coverage. Slit masks were created using the DEIMOS dsimulator slit-mask design software, which fills in the mask area to the extent possible with the highest priority input targets. This automatic selection was then adjusted by hand as appropriate. The remaining space on the slit masks was filled in with lower priority targets. The slit width for all masks was $0.7^{\prime \prime}$, and the minimum slit lengths were $\sim 5^{\prime \prime}$, depending slightly on the density of target stars.

In addition to the dwarf galaxy observations, we also obtained spectra of a radial velocity standard star, several telluric standards, and stars in the globular cluster NGC 1904 to serve as templates for cross-correlation with the dSph stars. More template observations (the globular cluster NGC 2419 and other radial velocity standards) were obtained during additional recent Keck DEIMOS observing runs, with identical observing setups (except for the slit width).

\subsection{Data Reduction}

The data were reduced using version 1.1.4 of the DEIMOS data reduction pipeline developed for the DEEP2 Galaxy Redshift Survey (M. C. Cooper 2007, private communication). Since this software was designed for faint, resolved galaxies, we modified the pipeline to optimize reductions for our relatively bright unresolved stellar targets. The main modifications were to change the cosmicray rejection algorithm and to allow alignment of individual twodimensional exposures in the spatial direction before co-adding. In addition, we modified the long-slit pipeline to allow proper reduction of very bright standard stars.

\subsection{Measurement of Radial Velocities}

We measure radial velocities by cross-correlating the observed science spectra with a set of high-S/N stellar templates. The stellar templates were observed with Keck DEIMOS using the same setup described above. Because template mismatch can result in significant velocity errors, we include a wide variety of stellar types and metallicities in our template library: giants of spectral type F8 III through M8 III, subgiants, and dwarf stars. In order to cover the range of metallicity expected in our low-luminosity dwarf galaxies, we also include several RGB and horizontal branch (HB) stars taken from observations of Galactic globular clusters. The stellar templates cover the metallicity range $[\mathrm{Fe} / \mathrm{H}]=-2.12$ to +0.11 . All science and template spectra are rebinned onto a common wavelength array with logarithmic wavelength bins of size $15 \mathrm{~km} \mathrm{~s}^{-1}$ pixel $^{-1}$, which is chosen to match the lowest spectral resolution present in the observed data.

We calculate and apply a telluric correction to each velocity measurement to account for velocity errors that result from miscentering an unresolved star within the slit. Following Sohn et al. (2007), we cross-correlate each science spectrum with a telluric template in the regions of the strong telluric absorption: 6860$6925,7167-7320,7593-7690$, and 8110-8320 ̊. The telluric template was created from the spectrum of a hot, rapidly rotating star (HR 1641, B3 V) that was allowed to drift perpendicularly across the slit (i.e., across the $0.7^{\prime \prime}$ dimension) during the exposure, simulating a source that uniformly fills the slit, and thus accurately reflects the mean integrated slit function. The mean telluric offset per mask ranged between -7 and $+2 \mathrm{~km} \mathrm{~s}^{-1}$, with a standard deviation within a mask of $3 \mathrm{~km} \mathrm{~s}^{-1}$. This correction is the velocity error caused by the mis-centering of the science star within the slit from, e.g., astrometry errors, or small mask rotation. Repeat observations of a number of stars on multiple masks demonstrate that the telluric correction reduces the mean absolute deviation between independent pairs of measurements from 4.6 to $3.8 \mathrm{~km} \mathrm{~s}^{-1}$, reduces the weighted standard deviation of the velocity differences between pairs of measurements from 5.6 to $4.2 \mathrm{~km} \mathrm{~s}^{-1}$, and improves the weighted mean difference from -2.0 to $-0.4 \mathrm{~km} \mathrm{~s}^{-1}$, indicating that the telluric correction is removing both random and systematic errors from the data.

We first calculate the telluric offset $\left(v_{\text {tell }}\right)$ and then determine radial velocities $\left(v_{\text {obs }}\right)$ for each science spectrum. In both cases, the template and science spectra are continuum subtracted; the template is then shifted and scaled to find the best fit in reduced- $\chi^{2}$ 

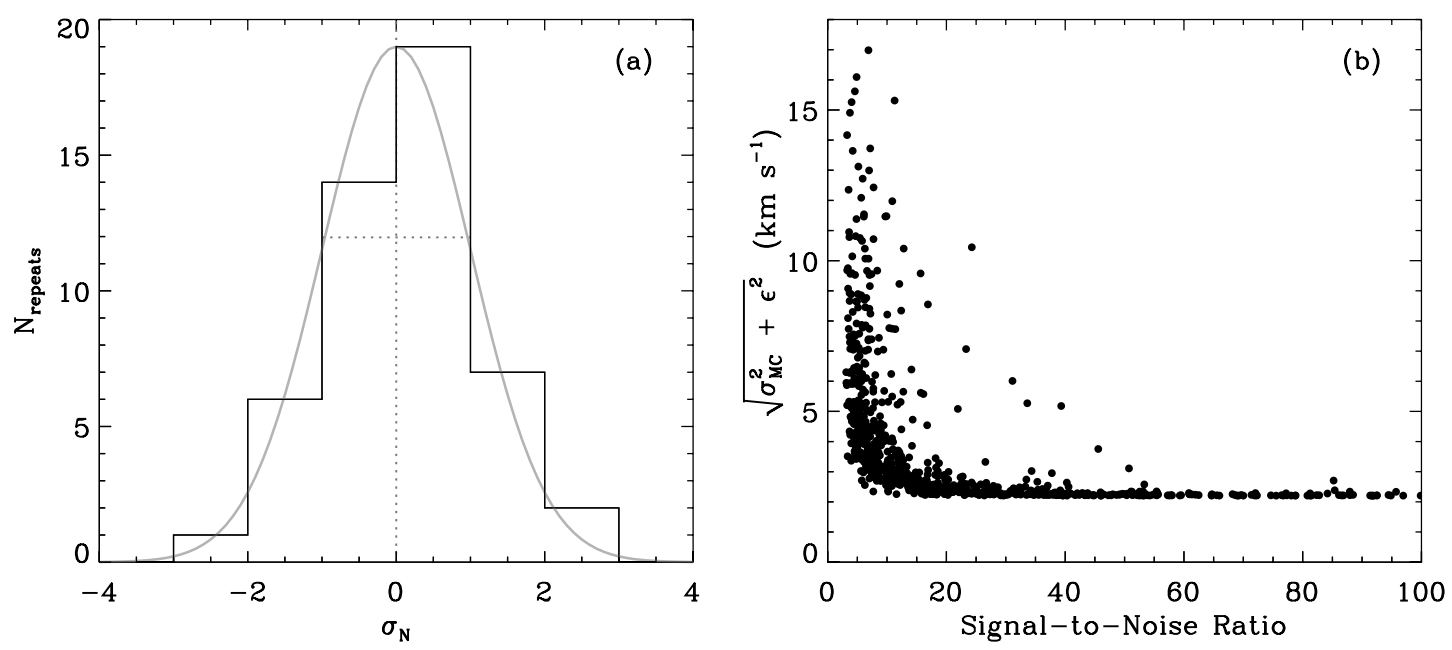

Fig. 1.- (a) Distribution of the normalized velocity error, $\sigma_{N}$ (as defined in eq. [1]), for 49 pairs of repeated independent velocity measurements. The best-fitting systematic error, $\epsilon=2.2 \mathrm{~km} \mathrm{~s}^{-1}$, is used to produce the unit Gaussian distribution shown by the curve and dotted lines. $(b)$ Combined random $\left(\sigma_{\mathrm{MC}}\right)$ and systematic velocity error for individual measurements plotted as a function of the mean per pixel $\mathrm{S} / \mathrm{N}$. These data include all of our science targets, but not the globular cluster and standard stars observed as spectroscopic templates. Points that fall far from the main locus are typically hot horizontal branch stars that lack the sharp spectral features present in the majority of giant and dwarf stars that comprise our sample. Note that five stars in the sample have velocity uncertainties larger than $18 \mathrm{~km} \mathrm{~s}^{-1}$, and 62 stars have $\mathrm{S} / \mathrm{N}$ greater than 100 , and are therefore not displayed in this plot; we chose the axis ranges so as to make the detailed distribution of uncertainties more visible. [See the electronic edition of the Journal for a color version of this figure.]

space. The final radial velocity $(v)$ is then: $v=v_{\text {obs }}-v_{\text {tell }}-v_{\text {hel }}$, where $v_{\text {hel }}$ is the heliocentric correction determined for each mask. All the radial velocities presented in this paper include a telluric and heliocentric correction.

The internal velocity dispersions of low-luminosity dwarf galaxies are, in many cases, of the same order as the DEIMOS velocity errors associated with individual measurements. In this regime, it is crucial to measure not only accurate velocities, but accurate velocity uncertainties. Underestimating (overestimating) the velocity uncertainties translates directly into larger (smaller) values of the inferred velocity dispersion using the methods described in $\S 3.2$. We determine our velocity error bars using a Monte Carlo bootstrap method, determine the contribution from systematic errors via repeat measurements of individual stars, and check the precision of these errors by comparing to higher spectral resolution data.

For the Monte Carlo method, noise is added to each pixel in the one-dimensional spectrum of each science observation based on the observed variance in that pixel. We assume the variance in each pixel is independent and distributed according to Poisson statistics. We then recalculate the velocity and telluric correction for this new spectrum using the routines above. Error bars are defined as the square root of the variance in the recovered mean velocity over 500 runs of the simulations. We next compare these Monte Carlo error estimates to the velocity differences between independent repeat measurements of individual stars. Since many of our DEIMOS masks covered overlapping sky areas, we placed 43 stars on two or more masks to obtain multiple independent velocity measurements. We note that one of these stars is likely an RR Lyrae variable ( $\S 3.5$ ) and remove it from the sample of repeated observations. We are left with 49 pairs of independent velocity measurements. The velocity difference between these independent observations samples the "true" error distribution. We define a normalized error $\left(\sigma_{N}\right)$ as the velocity difference between two independent measurements $\left(v_{1}, v_{2}\right)$, divided by the quadrature sum of all error contributions:

$$
\sigma_{N}=\frac{v_{1}-v_{2}}{\sqrt{\sigma_{\mathrm{MC} 1}^{2}+\sigma_{\mathrm{MC} 2}^{2}+2 \epsilon^{2}}},
$$

where the Monte Carlo errors $\left(\sigma_{\mathrm{MC} 1}, \sigma_{\mathrm{MC} 2}\right)$ on each measurement are combined in quadrature with an additional term, $\epsilon$, equal to the error contribution from systematics not accounted for in the Monte Carlo simulation. The $\sigma_{N}$ distribution should be a Gaussian of unit width. We therefore determine the unknown contribution from other systematic errors by fitting the parameter $\epsilon$ to produce a unit Gaussian distribution. In the left panel of Figure 1, the final $\sigma_{N}$ distribution is plotted for the best-fitting value of $\epsilon=2.2 \mathrm{~km} \mathrm{~s}^{-1}$. The final velocity errors used in our analysis are the quadrature sum of the Monte Carlo and systematic errors. In the right panel of Figure 1, we plot the final velocity errors as a function of the mean per pixel $\mathrm{S} / \mathrm{N}$ for all the individual stellar velocities presented in this paper. Stars that fall far from the main locus of points are typically hot horizontal branch stars (which have few sharp spectral features). The median velocity uncertainty for our sample of member stars in the ultra-faint dwarfs is $3.4 \mathrm{~km} \mathrm{~s}^{-1}$; including mask alignment stars and bright foreground stars that tend to have higher $\mathrm{S} / \mathrm{N}$, the median uncertainty for the entire data set is $2.7 \mathrm{~km} \mathrm{~s}^{-1}$.

To demonstrate our ability to accurately measure velocities and recover velocity dispersions, we compare our observations of the Galactic globular cluster NGC 2419 to higher spectral resolution Keck HIRES observations of the same cluster (P. Côté 2007 , private communication). We measure radial velocities for 26 stars between $1^{\prime}$ and $4^{\prime}$ of the cluster center. The HIRES data contain a similar number of stars in this region, although very few stars overlap between the two data sets. The HIRES spectrograph has a high spectral dispersion $\left(0.02 \AA\right.$ pixel $\left.^{-1}\right)$ and much more accurate individual velocity measurements $\left(\sim 0.95 \mathrm{~km} \mathrm{~s}^{-1}\right)$. We compute the recession velocity and velocity dispersion of NGC 2419 for both data sets using the maximum-likelihood technique described in $\S 3.2$. For the recession velocity, we measure $\langle\hat{u}\rangle_{\text {DEIMOS }}=-20.7 \pm 0.6 \mathrm{~km} \mathrm{~s}^{-1}$ compared to $\langle\hat{u}\rangle_{\mathrm{HIRES}}=$ $-21.2 \pm 0.5 \mathrm{~km} \mathrm{~s}^{-1}$, and for the velocity dispersion, $\sigma_{\text {DEIMOS }}=$ $2.3 \pm 0.4 \mathrm{~km} \mathrm{~s}^{-1}$ compared to $\sigma_{\text {HIRES }}=2.3 \pm 0.3 \mathrm{~km} \mathrm{~s}^{-1}$. The DEIMOS observations agree within the $1 \sigma$ limits of the more accurate HIRES measurements. Both sets of measurements agree with the published values for this cluster (Pryor \& Meylan 1993). While our NGC 2419 observations have somewhat higher S/Ns 
than those typical of our dwarf galaxy observations, this comparison demonstrates that we are able to reliably measure the kinematics in systems with extremely low velocity dispersions (smaller than expected for the dwarf galaxies). We also note that we measure a metallicity based on the Ca triplet lines ( $§ 2.4)$ for NGC 2419 of $[\mathrm{Fe} / \mathrm{H}]=-2.0$, and the data are consistent with no intrinsic metallicity spread within the cluster. The standard metallicity for this cluster is $[\mathrm{Fe} / \mathrm{H}]=-2.12$ (Harris 1996).

As a further test of our ability to measure reliable velocities, we compare our observations to high-resolution spectroscopy in UMa I by Kleyna et al. (2005). These authors presented Keck HIRES spectra of seven stars in the UMa I region (five members and two nonmembers). We re-observed all seven stars, with multiple measurements for two stars. ${ }^{4}$ We find excellent agreement between our measurements and those of Kleyna et al. for six of the stars (differences within the $1 \sigma$ uncertainties for five out of six and less than $1.7 \mathrm{~km} \mathrm{~s}^{-1}$ for all six); for star seven, both of our measurements are significantly discrepant with the Kleyna et al. velocity. Star 7 had the lowest S/N in Kleyna et al.'s observations, and they described the Ca triplet lines as "barely discernible above the noise." We conclude that either this star is a binary or variable star, or the velocity measured by Kleyna et al. (2005) is in error by $\sim 8 \mathrm{~km} \mathrm{~s}^{-1}$ (their estimated uncertainty is $5 \mathrm{~km} \mathrm{~s}^{-1}$ ).

Radial velocities were successfully measured for 1015 of the 1460 extracted spectra across the 18 observed science masks. This total includes 50 duplicate measurements of individual stars and 124 objects identified as galaxies or quasars. The latter objects will be very useful as background objects for proper motion studies and will be the subject of a future paper. The majority of spectra for which we could not measure a redshift did not have sufficient $\mathrm{S} / \mathrm{N}$. The fitted velocities are visually inspected to ensure the reliability of the measured redshift and the overall quality of the spectrum. The final sample of stellar radial velocities consists of 841 unique measurements across the eight target dwarf galaxies.

\subsection{Measurement of Equivalent Widths and Metallicities}

We estimate the metallicity $([\mathrm{Fe} / \mathrm{H}])$ of individual RGB stars in our target galaxies using the $\mathrm{Ca}$ II triplet absorption lines near $\lambda=8500 \AA$. We calculate the equivalent widths (EWs) of the three $\mathrm{Ca}$ II absorption lines using the line and continuum definitions of Rutledge et al. (1997b). The three EWs are combined into a single quantity as $\Sigma \mathrm{Ca}=0.5 \mathrm{EW}(8498 \AA)+1.0 \mathrm{EW}(8542 \AA)+$ $0.6 \mathrm{EW}(8662 \AA)$. We determine the error on this combined quantity with the Monte Carlo method described above. Added in quadrature to the Monte Carlo uncertainties is a systematic uncertainty of $0.3 \AA$, which we determined from repeat measurements as described in $\S 2.3$. We convert $\Sigma$ Ca into metallicity using the Rutledge et al. (1997a) empirical calibration relationship

$$
[\mathrm{Fe} / \mathrm{H}]=-2.66+0.42\left[\Sigma \mathrm{Ca}-0.64\left(V_{\mathrm{HB}}-V\right)\right] .
$$

The term $\left(V_{\mathrm{HB}}-V\right)$ is the magnitude difference between the horizontal branch and the observed star, and corrects for surface gravity effects. We assume an absolute magnitude for a metal-poor horizontal branch $M_{V, \mathrm{HB}}=0.88$ (Clem 2005), and calculate the apparent magnitude, $V_{\mathrm{HB}}$, using the distance modulus of each galaxy (see Table 1). The uncertainties in the distance moduli are included in the total metallicity uncertainties we derive. Note that assuming a single value for the horizontal branch magnitude in the possible presence of multiple stellar populations may add an addi-

\footnotetext{
4 Note that the SDSS DR2 coordinates given in Kleyna et al. (2005) for the target stars are up to $8^{\prime \prime}$ off from the true positions as given in the DR5 data or on Palomar Sky Survey plates.
}

tional $\sim 0.07$ dex to the metallicity uncertainties (Koch et al. 2006). We convert the SDSS $g$-band magnitudes into $V$ band using the photometric transformations of Blanton \& Roweis (2007) and reddening corrections from Schlegel et al. (1998). The Rutledge et al. calibration relation is derived for RGB stars in Milky Way globular clusters using the abundance scale of Carretta \& Gratton (1997), and while the calibration data only extend to $[\mathrm{Fe} / \mathrm{H}]=$ -2.1 it is reasonable to extrapolate the relation to the slightly lower metallicities found in some of our low-luminosity dwarfs (see $\S 3.4$ ). We restrict our metallicity analysis in $\S 3.4$ to only the RGB stars in the dwarf galaxies.

To remove foreground dwarf stars from the sample, we will use the equivalent width of the $\mathrm{Na}$ I $\lambda \lambda 8183,8195$ absorption lines, which are strongly dependent on surface gravity and temperature (Spinrad \& Taylor 1971; Schiavon et al. 1997). We measure the Na I equivalent width using the line and continuum definitions of Schiavon et al. (1997). Schiavon et al. show that the Na I EW is expected to be $1 \AA$ or greater in M-type dwarf stars, whereas this feature is much weaker in giant stars at the same temperature. Gilbert et al. (2006) have used this feature to successfully discriminate between dwarf and giant stars for a similar spectroscopic sample.

\section{RESULTS}

\subsection{Selection of Members}

We use two complementary techniques to determine which of the observed stars are members of the dwarf galaxies and which are foreground stars. The first method classifies stars based on objective criteria: velocity, distance from the fiducial RGB and HB tracks (corrected for foreground extinction using the reddenings from Schlegel et al. 1998 and for the distance of the galaxy), and the equivalent width of the $\mathrm{Na}$ I $\lambda 8190$ absorption lines (described above). The exact cutoffs for each of these parameters needed to be adjusted in a few cases, but in general we use a $3 \sigma$ cutoff in velocity (requiring a prior iteration to estimate the velocity dispersion), color-magnitude distance limits (defined as in $\S 2.1$ ) of 0.2 mag for RGB stars and 0.4 mag for HB stars, and a $\mathrm{Na}$ I equivalent width of less than $1.0 \AA$. Notable exceptions to these cutoffs include Coma Berenices, which is located so nearby that we detect a number of subgiants, blue stragglers, and mainsequence stars at $r>21.5$ - at these faint magnitudes we extend the allowed distance from the fiducial CMD track to 0.5 mag; and CVn I, which has a broad giant branch that also necessitates widening the cutoff distance from the RGB track. In addition, CVn I has so many member stars (214) that the presence of a $3 \sigma$ outlier is likely (and indeed we find one), so the velocity cutoff must be extended to $3.5 \sigma$, where there is only a $10 \%$ chance of finding a member star in our sample.

The second method is to examine individually the following properties of each star: velocity, location in the CMD, spatial position, fitted spectral type, metallicity, $\mathrm{Na}$ I equivalent width, and if necessary, the spectrum. Combining all of the available information about each star, and using thresholds similar to those described above (but less rigid), we classify each star as a likely member or nonmember "by eye." Both of these methods are similar in spirit to the techniques described by Gilbert et al. (2006) and Guhathakurta et al. (2006) for separating M31 red giants from foreground main-sequence stars, but without employing a full maximum-likelihood calculation, which is not necessary for these data because the $\mathrm{dSph}$ stars are more localized in parameter space than M31 halo stars are, and because of the higher S/N. In all cases, we find excellent agreement between the member samples identified with the two methods (with occasional threshold tweaks 

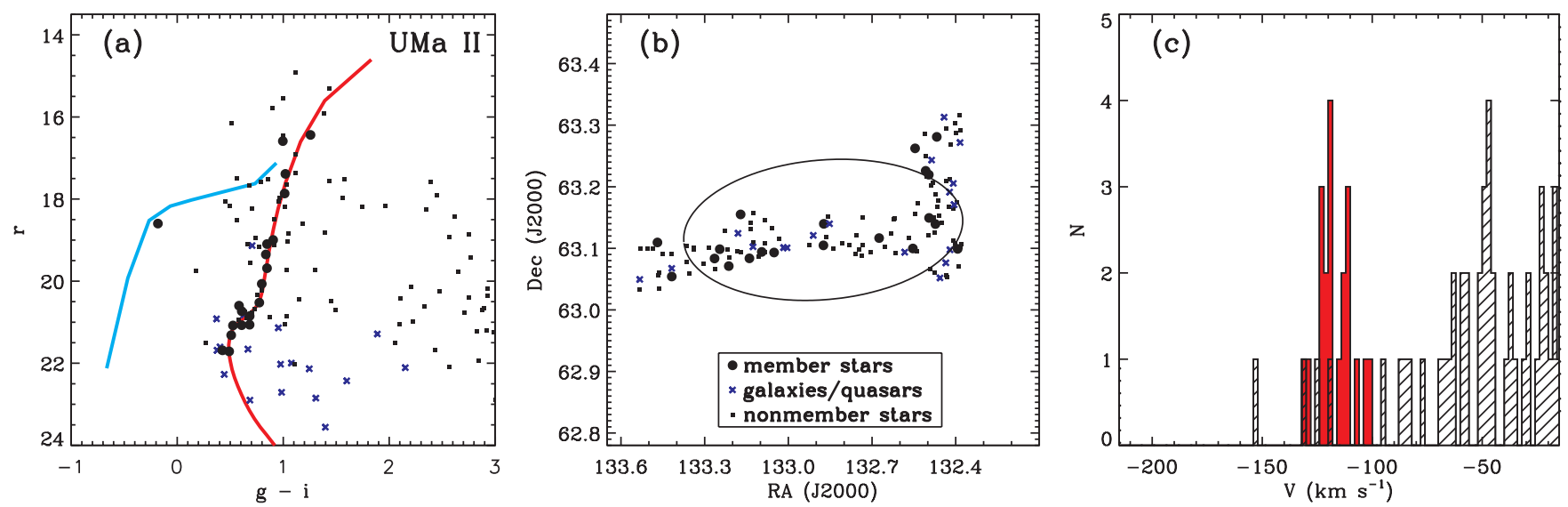

FIG. 2.- (a) Color-magnitude diagram of observed stars in Ursa Major II. The large black circles represent stars identified as radial velocity members of the galaxy, the small black dots represent stars identified as nonmembers, and the blue crosses are spectroscopically confirmed background galaxies and quasars. The red curve shows the location of the RGB, subgiant branch, and main-sequence turnoff populations in the globular cluster M92 and the blue curve shows the location of the horizontal branch of M13, both corrected for Galactic extinction and shifted to a distance of $32 \mathrm{kpc}$ (data from Clem 2005). (b) Spatial distribution of observed stars in Ursa Major II. Symbols are the same as in ( $a$ ) (the figure legend applies to both panels), and the ellipse represents the half-light radius of UMa II from Zucker et al. (2006b). (c) Velocity histogram of observed stars in Ursa Major II. Velocities are corrected to the heliocentric rest frame. The filled red histogram represents stars classified as members, and the hatched black-and-white histogram represents nonmembers. The velocity bins are $2 \mathrm{~km} \mathrm{~s}^{-1}$ wide.

required to produce a perfect match). A few of the galaxies contain questionable member stars that significantly affect the derived velocity dispersions; these cases will be discussed individually in $\S 3.5$. We display color-magnitude diagrams and spatial distributions for the observed stars in each dwarf galaxy in Figures 2-9.

\subsection{Central Velocity Dispersions}

Given the member samples selected in the previous subsection, we use the maximum-likelihood method described by Walker et al. (2006a) to calculate simultaneously the mean velocities and velocity dispersions of each galaxy. ${ }^{5}$ This method assumes that the observed velocity dispersion is the sum of the intrinsic galaxy dispersion and the dispersion produced by measurement errors, as well as that the velocity distribution is reasonably approximated by a Gaussian. The derived velocities and intrinsic velocity dispersions are displayed in Table 3. We find dispersions ranging from

\footnotetext{
${ }^{5}$ Note that the numerical values of the parameters $a$ and $b$ in eq. (9) of Walker et al. are negative, since they are proportional to the second derivatives of $\ln p$ evaluated at the maximum of the function. The uncertainties on the mean velocity and the velocity dispersion should therefore be defined as $d\langle\hat{u}\rangle=\sqrt{|a|}$ and $d \sigma=\sqrt{|b|}$ to avoid imaginary results.
}

$3.3 \pm 1.7 \mathrm{~km} \mathrm{~s}^{-1}$ for Leo IV to $7.6 \pm 0.4 \mathrm{~km} \mathrm{~s}^{-1}$ for Canes Venatici I.

We plot the stellar velocity dispersions as a function of absolute magnitude in Figure 10a. There is a significant correlation of velocity dispersion with absolute magnitude, with the more luminous galaxies $\left(M_{V} \lesssim-6\right)$ having larger dispersions of $\sim 7-8 \mathrm{~km} \mathrm{~s}^{-1}$ and the fainter galaxies $\left(M_{V} \gtrsim-6\right)$ exhibiting smaller dispersions of $\sim 4-5 \mathrm{~km} \mathrm{~s}^{-1}$. The four low-luminosity galaxies Coma Berenices, CVn II, Hercules, and Leo IV are the first galaxies to break the velocity dispersion "barrier" at $\sim 7 \mathrm{~km} \mathrm{~s}^{-1}$ that observations of the previously known dSphs had suggested (Gilmore et al. 2007). The unprecedentedly low velocity dispersions of these galaxies and the correlation with absolute magnitude down to such low luminosities demonstrate that if there is a floor on the masses of dSphs, it does not appear to have been reached yet.

The likely presence of unresolved binary stars in our stellar velocity sample may increase the measured velocity dispersion of our target galaxies due to binary orbital motion. Olszewski et al. (1996) simulated the effect of binaries on the velocity dispersions of the Draco and Ursa Minor dSphs with very similar sample sizes and velocity uncertainties as the present study. Assuming the binary fractions determined for Draco and Ursa Minor (which range
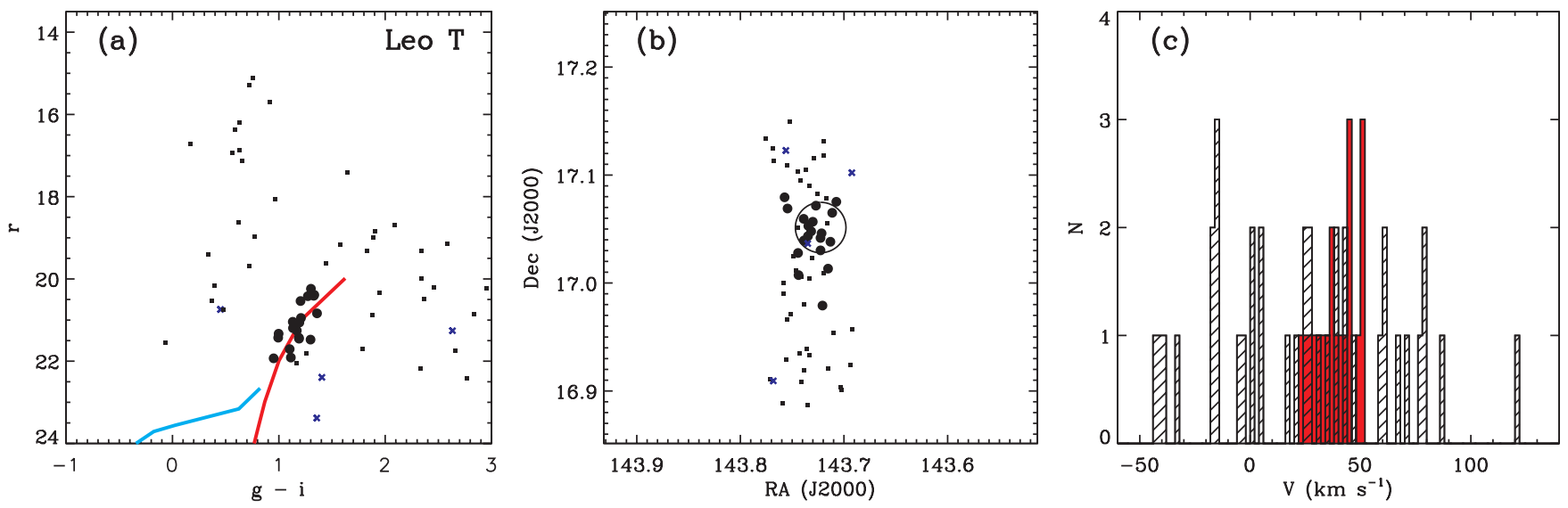

FIG. 3.- Same as Fig. 2, but for Leo T. 

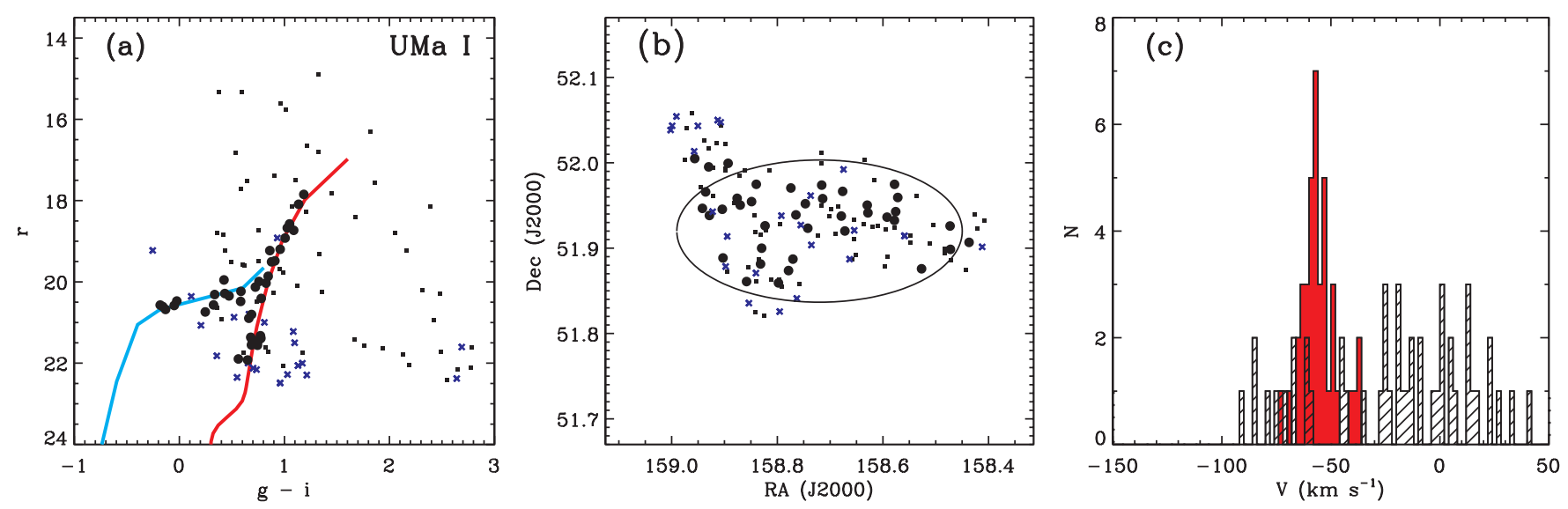

FIG. 4.-Same as Fig. 2, but for Ursa Major I.
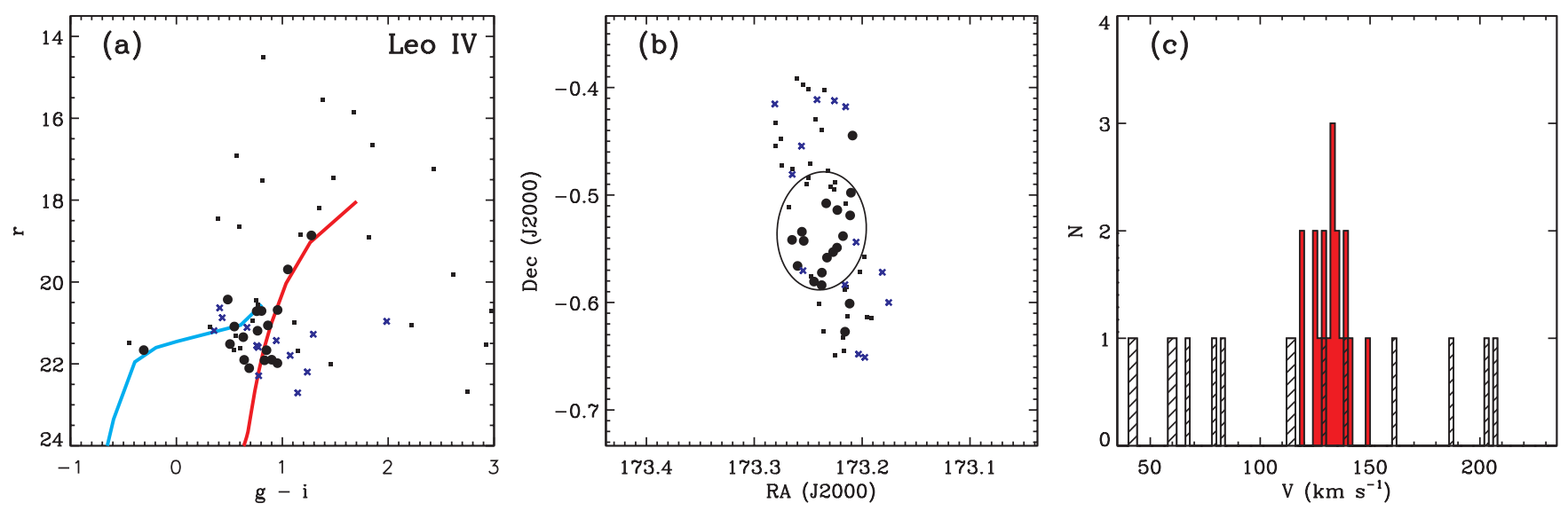

FIG. 5.- Same as Fig. 2, but for Leo IV.
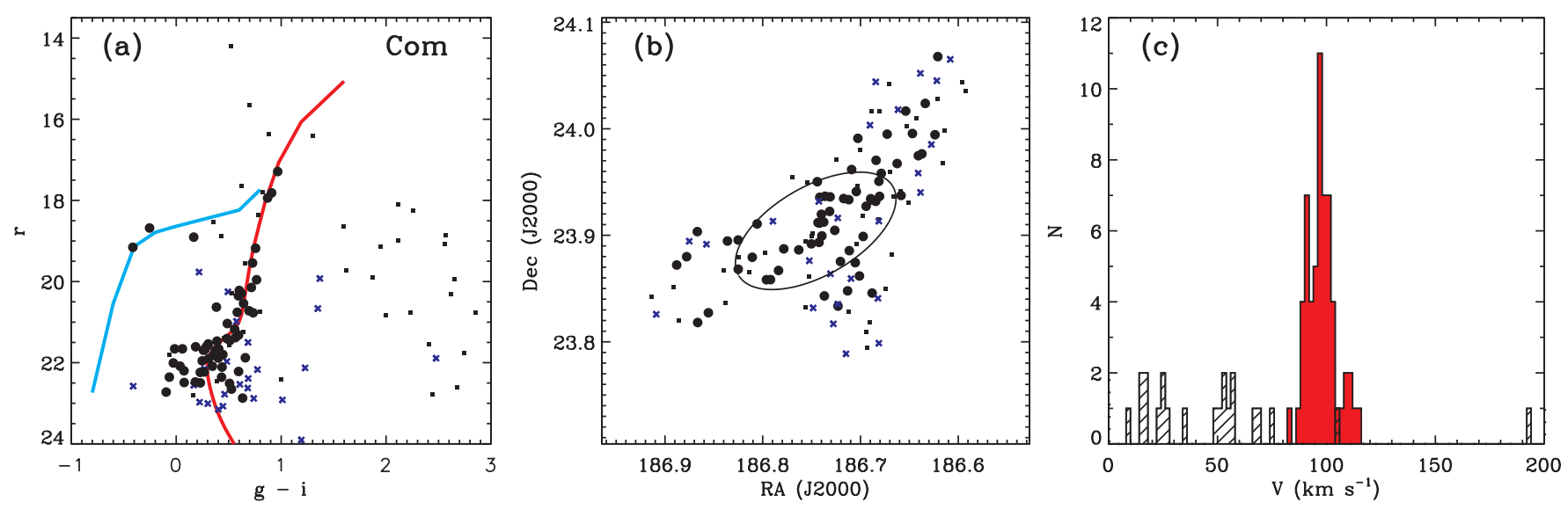

Fig. 6.-Same as Fig. 2, but for Coma Berenices. 

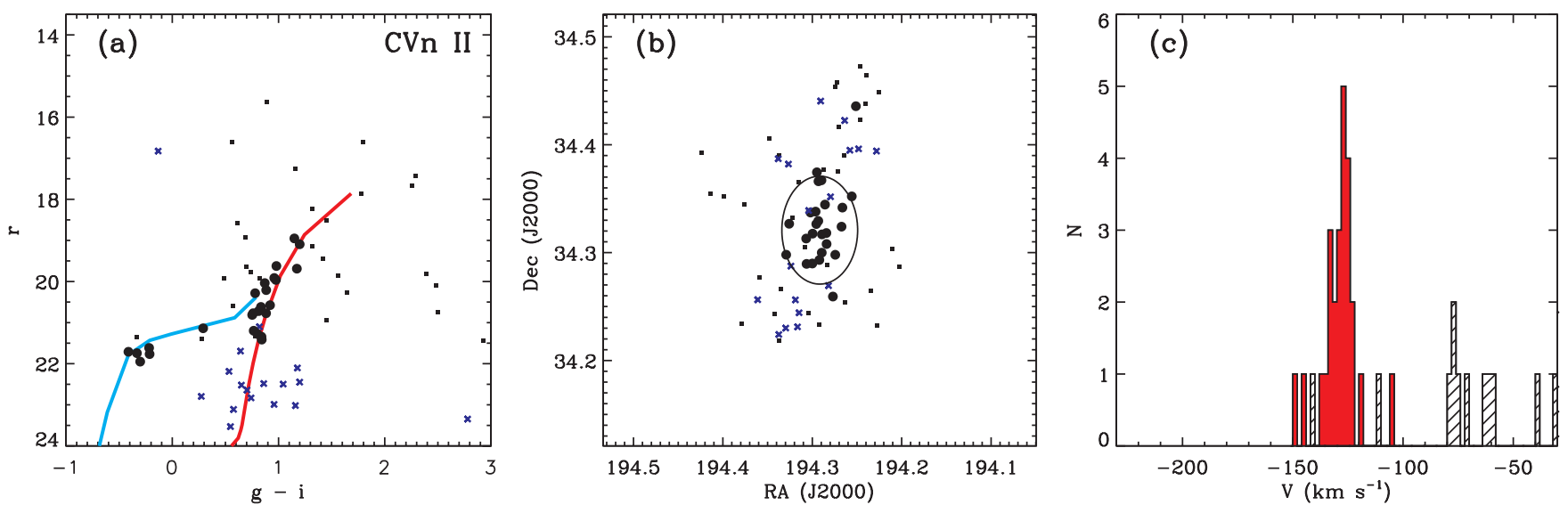

Fig. 7.- Same as Fig. 2, but for Canes Venatici II.
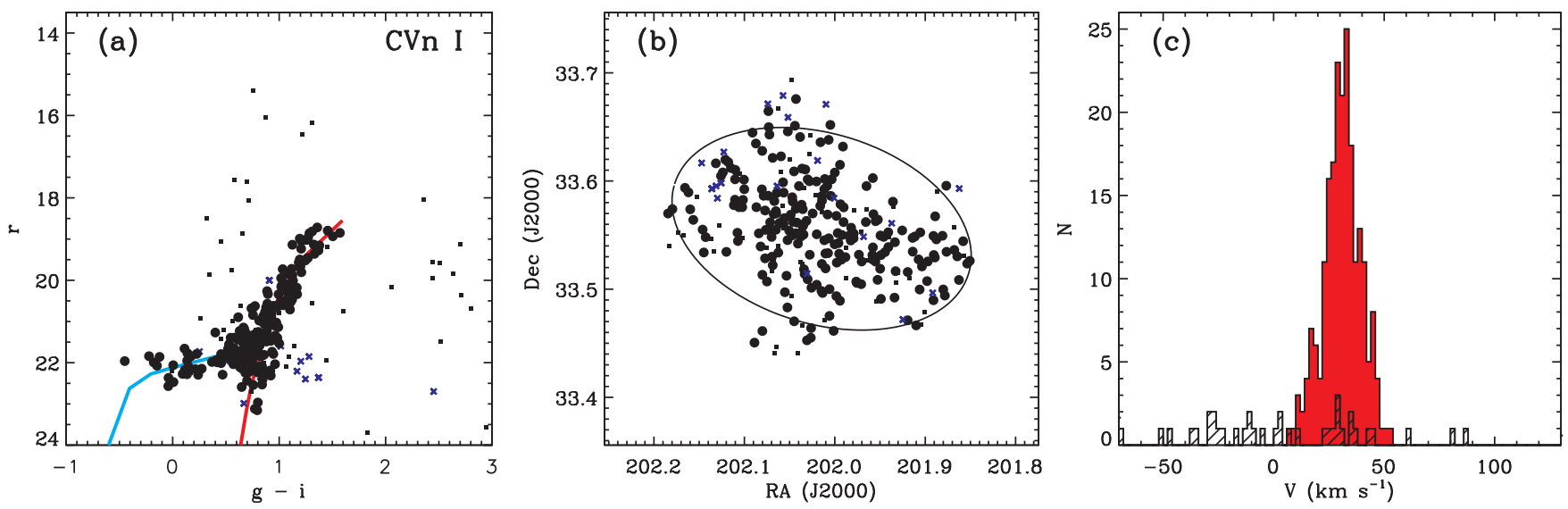

FIg. 8.- Same as Fig. 2, but for Canes Venatici I.
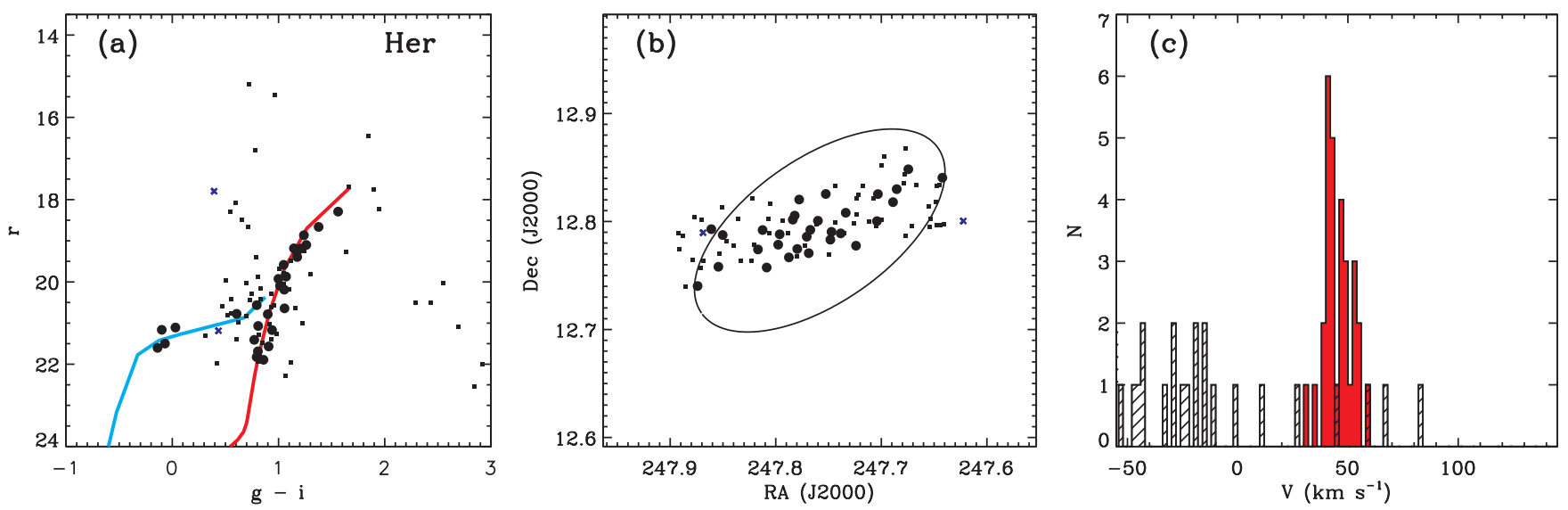

FIG. 9.- Same as Fig. 2, but for Hercules. 
TABLE 3

Radial Velocities and Velocity Dispersions

\begin{tabular}{|c|c|c|c|c|c|c|}
\hline Galaxy & $\begin{array}{c}\langle\hat{u}\rangle_{\mathrm{hel}} \\
\left(\mathrm{km} \mathrm{s}^{-1}\right)\end{array}$ & $\begin{array}{c}d\langle\hat{u}\rangle_{\text {hel }} \\
\left(\mathrm{km} \mathrm{s}^{-1}\right)\end{array}$ & $\begin{array}{c}\langle\hat{u}\rangle_{\mathrm{GSR}} \\
\left(\mathrm{km} \mathrm{s}^{-1}\right)\end{array}$ & $\begin{array}{c}\sigma \\
\left(\mathrm{km} \mathrm{s}^{-1}\right)\end{array}$ & $\begin{array}{c}d \sigma \\
\left(\mathrm{km} \mathrm{s}^{-1}\right)\end{array}$ & $\begin{array}{c}\text { Number of } \\
\text { Stars }\end{array}$ \\
\hline Ursa Major II ...................... & -116.5 & 1.9 & -33.4 & 6.7 & 1.4 & 20 \\
\hline Leo T................................... & 38.1 & 2.0 & -58.4 & 7.5 & 1.6 & 19 \\
\hline Ursa Major I ....................... & -55.3 & 1.4 & -7.1 & 7.6 & 1.0 & 39 \\
\hline 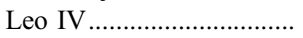 & 132.3 & 1.4 & 10.1 & 3.3 & 1.7 & 18 \\
\hline Coma Berenices ............... & 98.1 & 0.9 & 81.7 & 4.6 & 0.8 & 59 \\
\hline Canes Venatici II.............. & -128.9 & 1.2 & -95.5 & 4.6 & 1.0 & 25 \\
\hline Canes Venatici I................. & 30.9 & 0.6 & 77.6 & 7.6 & 0.4 & 214 \\
\hline 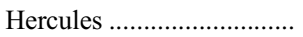 & 45.0 & 1.1 & 144.6 & 5.1 & 0.9 & 30 \\
\hline
\end{tabular}

between 0.2 and 0.3 for relevant binary periods), Olszewski et al. suggested that the velocity dispersion from binaries alone is on the order of $1.5 \mathrm{~km} \mathrm{~s}^{-1}$. Since it is possible that the binary fractions may be different in the lower luminosity galaxies we observed, we use this estimate only as a guide. For the highest velocity dispersion systems listed in Table 3, the effect of binaries is negligible. This result is consistent with conclusions from previous groups for other Local Group dSphs (Kleyna et al. 1999; Walker et al. 2006a). For our lowest dispersion system, Leo IV, the Olszewski et al. binary correction would reduce the measured dispersion from 3.3 to $2.9 \mathrm{~km} \mathrm{~s}^{-1}$. However, this difference is significantly smaller than our measurement uncertainty of $1.7 \mathrm{~km} \mathrm{~s}^{-1}$, so we do not correct our measured dispersions for the presence of binaries. Unless the binary star fraction in these ultra-low luminosity dwarfs is significantly larger than that of other dwarf galaxies, binaries do not significantly inflate the measured dispersions and inferred masses of even the lowest dispersion dwarf galaxies in our sample.

\subsection{Total Masses}

The process of determining the total mass of a dwarf spheroidal galaxy from the velocities of a relatively modest sample of stars that are probably located well inside the virial radius of the galaxy's dark matter halo is fraught with difficulty. The standard technique in the literature is to assume that (1) the galaxy is spherical; (2) the galaxy is in dynamical equilibrium; (3) the galaxy has an isotropic velocity dispersion; and (4) the light distribution of the galaxy traces its mass distribution. All four of these assumptions may be false in reality, especially for the ultra-faint dwarfs that are the subject of this paper. SDSS photometry and follow-up imaging reveal that most of the dwarfs are elongated, demonstrating that they are not spherically symmetric systems and probably do not have isotropic velocity dispersion tensors. Several of the dwarfs also appear irregular, opening up the possibility that their structure has been significantly affected by the tidal field of the Milky Way. However, these apparently irregular isodensity contours could also be the result of the extremely low surface densities of the galaxies, which make their stellar distributions difficult to determine accurately. Finally, the nearly flat velocity dispersion profiles observed in all of the dSphs where spatially resolved kinematics are available indicate that light does not trace mass (Walker et al. 2006a; Wu 2007). Despite these objections, the samples of stars in the ultra-faint dwarfs that are spectroscopically accessible with current instruments are so small that more sophisticated analyses are not possible (with the exception of CVn I, which will be discussed in more detail in a future paper). We therefore use the method of Illingworth (1976) to estimate total masses for the observed galaxies:

$$
M_{\mathrm{tot}}=167 \beta r_{c} \sigma^{2},
$$

where $\beta$ is a parameter that depends on the concentration of the system and is generally assumed to be 8 for dSphs (Mateo 1998), $r_{c}$ is the King (1962) profile core radius, and $\sigma$ is the observed central velocity dispersion. For most of the new dwarfs, only Plummer
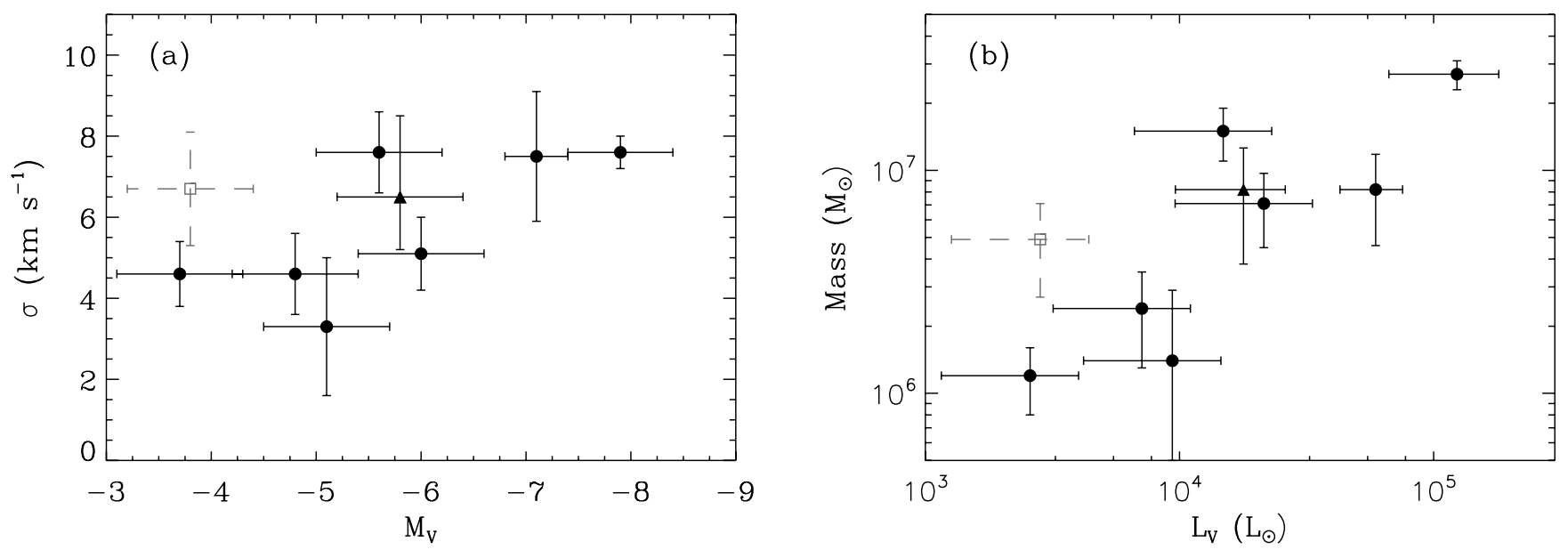

FIG. 10.- (a) Velocity dispersion as a function of absolute magnitude for the ultra-faint dwarfs. The filled black symbols represent the gravitationally bound dwarfs and the open gray symbol represents UMa II, which is thought to be tidally disrupted (see §3.6). Circles are ultra-faint dwarfs in this sample and the triangle is the Boötes dSph (Martin et al. 2007). (b) Dynamical mass as a function of total $V$-band luminosity. Symbols are the same as in $(a)$. The ultra-faint dwarf galaxies clearly display a trend in which the more luminous galaxies have larger velocity dispersions and correspondingly larger masses. Perhaps surprisingly, there appears to be a simple power-law relationship between mass and luminosity. 
TABLE 4

Masses, Mass-to-Light Ratios, and Metallicities

\begin{tabular}{|c|c|c|c|c|}
\hline Galaxy & $\begin{array}{l}\text { Mass } \\
\left(M_{\odot}\right)\end{array}$ & $\begin{array}{c}M / L_{V} \\
\left(M_{\odot} / L_{\odot}\right)\end{array}$ & {$[\mathrm{Fe} / \mathrm{H}]$} & $\sigma_{[\mathrm{Fe} / \mathrm{H}]}$ \\
\hline Ursa Major II $^{\mathrm{a}}$. & $(4.9 \pm 2.2) \times 10^{6}$ & $1722 \pm 1226$ & $-1.97 \pm 0.15$ & 0.28 \\
\hline 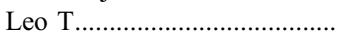 & $(8.2 \pm 3.6) \times 10^{6}$ & $138 \pm 71$ & $-2.29 \pm 0.10$ & 0.35 \\
\hline 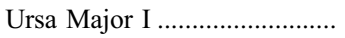 & $(1.5 \pm 0.4) \times 10^{7}$ & $1024 \pm 636$ & $-2.06 \pm 0.10$ & 0.46 \\
\hline 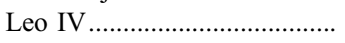 & $(1.4 \pm 1.5) \times 10^{6}$ & $151 \pm 177$ & $-2.31 \pm 0.10$ & 0.15 \\
\hline Coma Berenices ....................... & $(1.2 \pm 0.4) \times 10^{6}$ & $448 \pm 297$ & $-2.00 \pm 0.07$ & 0.00 \\
\hline Canes Venatici II..................... & $(2.4 \pm 1.1) \times 10^{6}$ & $336 \pm 240$ & $-2.31 \pm 0.12$ & 0.47 \\
\hline Canes Venatici I........................ & $(2.7 \pm 0.4) \times 10^{7}$ & $221 \pm 108$ & $-2.09 \pm 0.02$ & 0.23 \\
\hline 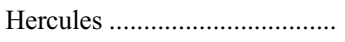 & $(7.1 \pm 2.6) \times 10^{6}$ & $332 \pm 221$ & $-2.27 \pm 0.07$ & 0.31 \\
\hline
\end{tabular}

${ }^{\text {a }}$ UMa II may be a tidally disrupted remnant, which would artificially inflate its mass and mass-to-light ratio.

(half-light) radii rather than King core radii are available in the literature, but we can use the fact that $r_{c}=0.64 r_{\text {Plummer }}$ to estimate the King radii. The radii and luminosities we have assumed for these calculations are given in the Appendix. Our derived masses for each galaxy are listed in Table 4 and plotted in Figure $10 b$. We note that objects in the bottom left corner of the plot are both the least massive and least luminous known galactic systems.

The ultra-faint Milky Way satellites have masses ranging from just over $10^{6} M_{\odot}$ (Coma Berenices) up to $2.8 \times 10^{7} M_{\odot}$ (Canes Venatici I). Not surprisingly, CVn I, which is nearly as bright as previously known dSphs such as Ursa Minor and Draco, has a mass that is similar to those of the original Milky Way dSphs. Combining the measured masses with the absolute magnitudes listed in Table 1, we can calculate $V$-band mass-to-light ratios, which are presented in Table 4 . The new dwarfs continue the trend of an anticorrelation between luminosity and $M / L$ that has been known for many years (e.g., Mateo et al. 1993), reaching mass-tolight ratios of $\sim 1000$ in $V$-band solar units. Although the uncertainties on the mass-to-light ratios are substantial, owing primarily to the poorly known luminosities of the ultra-faint dwarfs, it is clear that all of these galaxies have quite large mass-to-light ratios. The existence of galaxies with similar properties to these was predicted recently by Ricotti \& Gnedin (2005) and Read et al. (2006) but the measured masses seem to be in better agreement with the models of Ricotti \& Gnedin (2005).

\subsection{Metallicities}

The mean stellar metallicity of a galaxy reflects the enrichment history of the interstellar medium at the time the stars were formed. We determine the mean metallicity, $[\mathrm{Fe} / \mathrm{H}]$, for the new dwarf galaxies based on the $\mathrm{Ca}$ II triplet equivalent width ( $\S 2.4)$. While we can reliably measure equivalent widths for the majority of our target stars, the Rutledge et al. (1997a) empirical calibration that we use to convert to $[\mathrm{Fe} / \mathrm{H}]$ is valid only for RGB stars. We therefore only include stars brighter than $M_{V}=+1.5$ and redder than $(g-r)>0.3$ (to avoid HB stars) in the metallicity analysis. We determine the mean metallicity and metallicity spread using the maximum-likelihood technique described in $\S 3.2$. While the metallicity distributions are not necessarily Gaussian, as the maximumlikelihood calculation assumes, we find that the mean and median of the observed metallicity distributions give similar results. We run the maximum-likelihood algorithm twice, rejecting $3 \sigma$ outliers on the second run. The mean metallicities and metallicity spreads are listed in Table 4. We find metallicities ranging from $[\mathrm{Fe} / \mathrm{H}]=$ $-1.97 \pm 0.15$ for UMa II down to $[\mathrm{Fe} / \mathrm{H}]=-2.31$ for $\mathrm{CVn}$ II and Leo IV. We note that several of our galaxies have mean metallicities equal to those of the most metal-poor globular clusters and lower than those of other dwarf galaxies (Harris 1996; Mateo
1998), making them, along with the Boötes dSph (Muñoz et al. 2006a), the most metal-poor stellar systems known.

A strong correlation exists between the total luminosity of dwarf galaxies in the Local Group and their stellar metallicities (Mateo 1998; Grebel et al. 2003). In comparison, Galactic globular clusters follow no such relationship (Harris 1996). In Figure 11, we show that all but the two faintest of the ultra-low luminosity galaxies follow the luminosity-metallicity relationship defined by the more luminous dSphs. The two galaxies deviating from this relationship are UMa II and Com. These are the nearest as well as the lowestluminosity objects in our sample, and both galaxies (particularly UMa II) show a variety of evidence suggesting that they are undergoing tidal disruption by the Milky Way. As discussed in $\S 3.6$, we interpret the high metallicities in these two objects as evidence that their formation mass may have been significantly larger than their present mass.

The ultra-low luminosity galaxies extend the luminositymetallicity relation in the Local Group by an additional 4 mag to $M_{V}=-4.8$. The location of these seven objects (including Boötes) on the same relationship defined by brighter dSphs is significant. It suggests that the stars formed in these galaxies are connected to the present mass of the galaxy and argues against significant tidal stripping, unless the amount of mass stripped from each galaxy approximately preserved the relative ordering of dwarf masses. We also measure significant internal metallicity spreads, $\sigma_{[\mathrm{Fe} / \mathrm{H}]}$, up to 0.5 dex in several ultra-low luminosity dwarfs, as listed in Table 4. This suggests that stars formed in multiple star formation episodes, rather than a single burst, and firmly distinguishes these faint dwarfs from globular clusters, which do not contain mixed stellar populations. This is clearly the case for Leo T, which shows evidence for multiple stellar populations from its colormagnitude diagram (Irwin et al. 2007). However, a metallicity spread is the only evidence of multiple star formation episodes in the other dwarfs. Further investigation into the detailed abundances of these will provide a much clearer picture of star formation in these low-mass objects.

\subsection{Comments on Individual Galaxies}

Ursa Major II. - UMa II is one of the hardest galaxies to identify based on its signature in the velocity histogram (see Fig. 2c), but a clear peak at $-117 \mathrm{~km} \mathrm{~s}^{-1}$ emerges once the foreground dwarf stars are screened out by their $\mathrm{Na}$ I equivalent widths. Our measured velocity and velocity dispersion are in good agreement with those of Martin et al. (2007). We identify 20 member stars in UMa II out of 236 targeted sources, which represents our lowest detection rate for any of the galaxies. However, this is at least partly a result of our attempt to focus on stars in the outlying clumps noted by Zucker et al. (2006b) rather than the main body of the 


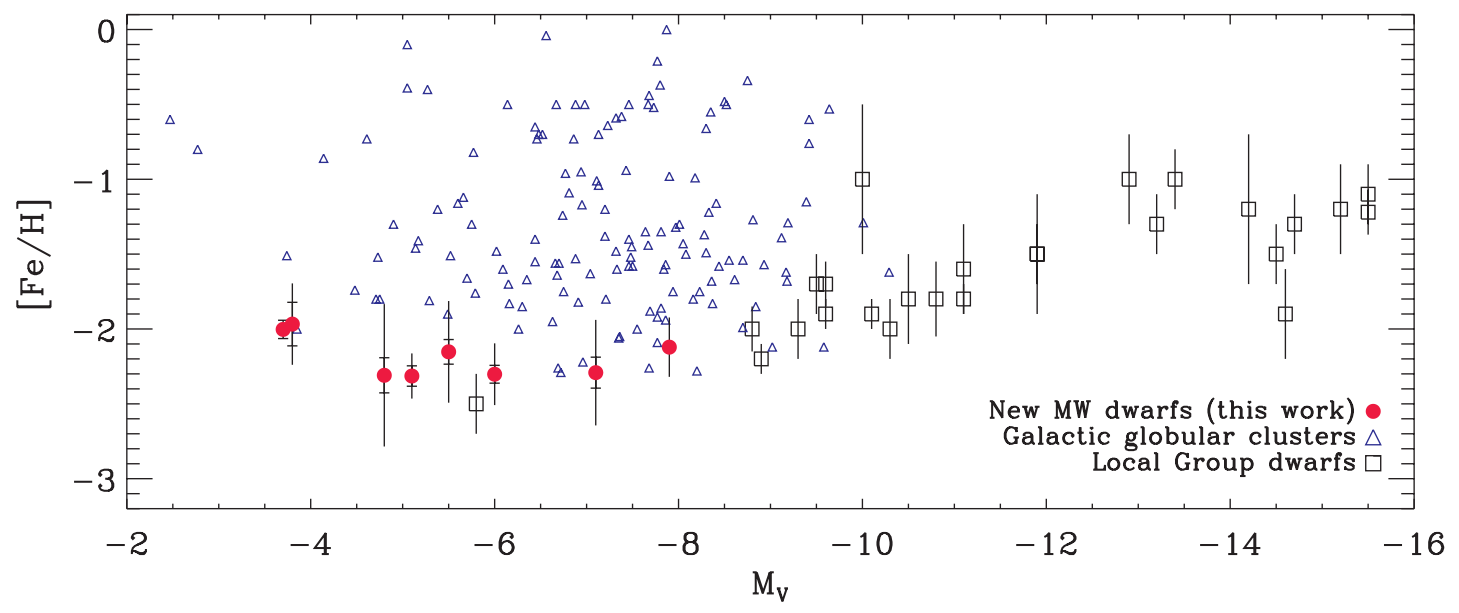

FIg. 11.-Metallicity-luminosity relationship for dwarf galaxies in the Local Group. The new ultra-faint galaxies (red circles) follow the trend of decreasing metallicity with luminosity set by more luminous dwarf galaxies (black squares). The two lowest-luminosity objects (UMa II and Com) show possible evidence of tidal stripping. In comparison, Galactic globular clusters (blue triangles) do not follow any luminosity-metallicity relationship. Data for luminous dwarf galaxies are from Mateo (1998), Galactic globular clusters from Harris (1996), and the ultra-low luminosity dwarf Boötes (open square at $M_{V}=-5.8$ ) from Muñoz et al. (2006a). Martin et al. (2007) find a somewhat higher metallicity for Boötes of $[\mathrm{Fe} / \mathrm{H}]=-2.1$. The smaller horizontal bars on our galaxy measurements represent the uncertainty in the mean metallicity; internal metallicity spreads are indicated by the larger vertical bars. The ultra-low luminosity dwarfs are among the most metal-poor stellar systems in the known universe.

dwarf. There are two additional stars we classify as nonmembers that could in fact be associated with UMa II. One of these stars has a velocity of $-95.6 \pm 2.3 \mathrm{~km} \mathrm{~s}^{-1}$, just over $3 \sigma$ away from the systemic velocity; including this star as a member would increase the velocity dispersion of UMa II to $8.2 \pm 1.6 \mathrm{~km} \mathrm{~s}^{-1}$. The other candidate member, SDSS J084947.6+630830, was observed on both the first and third nights of our run and shows a velocity shift of $52.5 \mathrm{~km} \mathrm{~s}^{-1}$ while also changing spectral type from a $\mathrm{K}$ giant to a horizontal branch star. We suspect that this star is an RR Lyrae variable, and its mean velocity and apparent magnitude suggest that it is plausibly associated with UMa II, but we must exclude it from our velocity dispersion calculation because of its large velocity variability. Future observations of this star could provide improved constraints on the distance of UMa II.

UMa II is a clear outlier from the $M_{V}-\sigma$ trend defined by the other galaxies in Figure $10 a$, with a dispersion of $6.7 \mathrm{~km} \mathrm{~s}^{-1}$ despite its incredibly low luminosity. The irregular appearance, proximity to the Milky Way, and low luminosity of UMa II led Zucker et al. (2006b) to suggest in their discovery paper that this galaxy might be in the process of tidal disruption. Belokurov et al. (2007a) and Fellhauer et al. (2007) have argued that UMa II is the progenitor of the recently discovered stellar tidal stream known as the Orphan Stream. In $\S 3.6$, we add additional kinematic and abundance evidence supporting the hypothesis that UMa II is a tidally disrupting satellite that may be associated with the Orphan Stream.

Leo T.-Leo T is unique among the new dwarfs in that it contains gas $\left(M_{\mathrm{H}} / M_{\mathrm{star}} \sim 1\right)$ and has formed stars in the relatively recent past (Irwin et al. 2007). We measure a mean velocity of $38.1 \pm 2.0 \mathrm{~km} \mathrm{~s}^{-1}$ and a stellar velocity dispersion of $7.5 \pm$ $1.6 \mathrm{~km} \mathrm{~s}^{-1}$, in excellent agreement with the $\mathrm{H}$ I velocity and gas velocity dispersion measured by Ryan-Weber et al. (2007). We do not detect any evidence for a cold stellar population to match the cold gas component at the center of the galaxy, but our sample of 19 member stars is not large enough for a significant detection of such a component. One would also only expect the youngest blue stars, which are not sampled by our observations, to have kinematics similar to the cold gas. Leo $\mathrm{T}$ is now one of very few dwarf galaxies that have well-measured kinematics from both the stars and the gas, and the agreement between the two indicates that the gas is accurately tracing the gravitational potential of the galaxy. In such a small system, many other effects could contribute to the velocity dispersion of the gas, but those contributions appear not to be significant. If this result also applies to other dwarfs, then $\mathrm{H}$ I kinematics can be used to measure their masses reliably, which is useful because in many cases the gas extends to larger radii than the stars do.

Ursa Major I.-Kleyna et al. (2005) reported Keck HIRES spectra of five UMa I member stars, obtaining a systemic velocity of $-52.45 \pm 4.27 \mathrm{~km} \mathrm{~s}^{-1}$ and a velocity dispersion of $9.3_{-1.2}^{+11.7} \mathrm{~km} \mathrm{~s}^{-1}$. We reobserved all seven of the stars from the Kleyna et al. sample (including the two nonmembers) and find excellent agreement on individual velocity measurements as discussed in $\S 2.3$. With our larger sample of 39 member stars, our mean velocity for UMa I is in good agreement with that of Kleyna et al., but our dispersion is somewhat lower than they measure (the disagreement is at less than $95 \%$ confidence). We rule out the extremely high velocity dispersions of up to $\sim 20 \mathrm{~km} \mathrm{~s}^{-1}$ allowed by the Kleyna et al. data. Using the same luminosity for UMa I that Kleyna et al. assumed $\left(M_{V}=-6.75\right.$ from Willman et al. 2005a), we naturally find a somewhat lower mass-to-light ratio (355 \pm $220 M_{\odot} / L_{\odot}$ ) than they calculate, but with the revised magnitude of $M_{V}=-5.5$ measured by Belokurov et al. (2006) $M / L$ becomes significantly larger. Our derived velocity dispersion is significantly lower than that obtained by Martin et al. (2007), which may indicate that their uncertainties have been underestimated (see $\S 2.3$ ). We do not detect any evidence for the kinematically cold component $\left(\sigma<3.4 \mathrm{~km} \mathrm{~s}^{-1}\right)$ suggested by Martin et al., despite a sample of stars that is a factor of $\sim 2$ larger.

UMa I lacks a published distance uncertainty. Willman et al. (2005a) estimated a distance of $100 \mathrm{kpc}$ from comparisons with the CMD of Sextans and theoretical isochrones. We use $\chi^{2}$ fits of the M92 RGB and M13 HB fiducial tracks to our sample of radial velocity member stars to measure a more accurate distance modulus for UMa I of $20.13_{-0.17}^{+0.18} \mathrm{mag}$, corresponding to a distance of $106_{-8}^{+9} \mathrm{kpc}$. We increase the assumed absolute magnitude of UMa I to $M_{V}=-5.6$ to compensate for this slight increase in distance.

Leo IV.-With only one slit mask devoted to it, and a total exposure time of less than an hour, Leo IV is the least well-studied galaxy in our sample. It also appears to have the smallest velocity dispersion, although with only 18 member stars and larger-thanaverage uncertainties on many of them, the uncertainty on the 
dispersion is significant. The dispersion of Leo IV also depends critically on our assumptions about membership. Two candidate member stars that we have rejected would significantly influence its properties if they were included. One of these stars, although it is located just above blue end of the horizontal branch, has a velocity of $v=160.1 \pm 5.9 \mathrm{~km} \mathrm{~s}^{-1}$ that is well beyond the $3 \sigma$ velocity range for the galaxy. Adding this star as a member would dramatically increase the velocity dispersion to $6.4 \pm 2.0 \mathrm{~km} \mathrm{~s}^{-1}$. We therefore reject this star as a nonmember (or possible binary system). The second star presents a more ambiguous case. It has a velocity of $120.2 \pm 2.8 \mathrm{~km} \mathrm{~s}^{-1}$, which is consistent with membership. It is located outside the half-light radius, but is close to two other member stars. However, inspection of the spectrum reveals that the Ca triplet lines for this star appear to be double peaked, and that a more appropriate velocity for this star may be $\sim 129 \mathrm{~km} \mathrm{~s}^{-1}$. Given that this star may be a binary and that its true velocity is uncertain, we consider the safest approach to be removing it from the sample. If the star is included with a velocity of $120.2 \mathrm{~km} \mathrm{~s}^{-1}$ the dispersion of Leo IV would be $5.0 \pm 1.4 \mathrm{~km} \mathrm{~s}^{-1}$; if the star's velocity is $129 \mathrm{~km} \mathrm{~s}^{-1}$ the effect on the velocity dispersion is negligible.

Coma Berenices.-Coma Berenices has the lowest luminosity of the new Milky Way satellites, and is located firmly in the low velocity dispersion half of the sample. One of the 59 assumed member stars has a velocity just outside the $3 \sigma$ limit, at $v=$ $83.1 \pm 2.8 \mathrm{~km} \mathrm{~s}^{-1}$. This star is located $\sim 0.3 \mathrm{mag}$ away from the blue edge of the subgiant branch, and could be an evolved blue straggler in Com. On the other hand, if we reject this star from the sample as a foreground contaminant, the velocity dispersion of Com declines to $3.8 \pm 0.8 \mathrm{~km} \mathrm{~s}^{-1}$. Com is unique among the ultra-faint dwarfs in that its member stars span a wide range of $g-i$ colors near the main-sequence turnoff (MSTO), although only UMa II is nearby enough to detect such stars among the other galaxies. The photometric uncertainties are not large enough to account for this spread. The stars on the blue side of the MSTO could be blue stragglers, but the presence of a few similarly situated stars on the red side suggests that we might instead be seeing the effects of multiple stellar populations with different ages (and hence MSTO luminosities and colors) in Com. Although it appears in Figure $6 c$ that there may be velocity substructure in Com, this is partly a result of the chosen velocity binning, and a twosided Kolmogorov-Smirnov (KS) test indicates that the observed velocity distribution is consistent with a Gaussian at the $17 \%$ confidence level.

In terms of its luminosity, radius, and proximity to the Milky Way, Com is most similar to UMa II among all of the ultra-faint satellites. Accordingly, we consider the possibility that, like UMa II, Com is in the process of being tidally disrupted. We discuss the available evidence for and against disruption $n \oint 3.6$, but conclude that Com is more likely to be a bound, dark matter dominated object.

Canes Venatici II.- CVn II is a very faint and very compact dwarf with a low mass. We have rejected one star as a member that is located well outside the half-light radius and has a velocity that is almost $4 \sigma$ away from the mean velocity. If we instead opt to include this star, the velocity dispersion of the galaxy increases somewhat to $5.1 \pm 1.1 \mathrm{~km} \mathrm{~s}^{-1}$.

Canes Venatici I.-Along with Leo T, CVn I is the only other of the new dwarfs to display a broad RGB, indicating a significant spread in metallicity and/or age among its stellar population. For Leo T, such a result is not surprising because young stars are visible in its CMD (Irwin et al. 2007) and it still contains gas, but it is somewhat less expected in a tiny, gas-poor system like CVn I. It is not currently understood how low-mass dSphs managed to hold on to enough gas to form stars over an extended period of time (although see, e.g., Ricotti \& Gnedin 2005; Marcolini et al. 2006), but the same phenomenon is observed in all of the more luminous Milky Way dSph satellites. In this sense, CVn I may have more in common with the previously known dSphs (it approaches the lower bound of their luminosities) than it does with its ultra-faint SDSS cousins (which have much lower luminosities). While other dwarfs in our sample show evidence for metallicity spreads, CVn I and Leo T-the two brightest of the ultra-faint dwarfs, and two of the three most massive (along with UMa I) are the only two with RGB spreads as well. This result suggests that the critical mass and luminosity for dwarf galaxies to maintain the ability to form multiple stellar populations are $M_{V} \lesssim-7$ and $M \gtrsim 10^{7} M_{\odot}$ (although see $\S 3.4$ ).

Ibata et al. (2006) measured the velocities of 44 stars in CVn I and reported two distinct kinematical components to the galaxy: a centrally concentrated metal-rich population with a velocity dispersion of less than $1.9 \mathrm{~km} \mathrm{~s}^{-1}$ at $99 \%$ significance and a more extended metal-poor component with a velocity dispersion of $13.9_{-2.5}^{+3.2} \mathrm{~km} \mathrm{~s}^{-1}$. Our much larger sample of $214 \mathrm{CVn}$ I member stars does not reveal any trace of this dichotomy. Dividing our sample in half using the same metal-rich/metal-poor cutoff as Ibata et al. ( $[\mathrm{Fe} / \mathrm{H}]=-2.0)$, we find $\sigma=8.1 \pm 0.8 \mathrm{~km} \mathrm{~s}^{-1}$ for the metal-rich stars and $\sigma=7.2 \pm 0.5 \mathrm{~km} \mathrm{~s}^{-1}$ for the metal-poor stars. Even limiting the metal-rich selection to the most metal-rich $\sim 10 \%$ of the stars (in case the population detected by Ibata et al. [2006] represents only a small fraction of the overall stellar population) does not reveal any evidence for a cold component. We also do not detect any tendency for the metal-rich stars to be more centrally concentrated in the galaxy than the metal-poor stars. Finally, we measure a mean velocity of $30.9 \pm 0.6 \mathrm{~km} \mathrm{~s}^{-1}$, inconsistent with the value of $\sim 24.5 \mathrm{~km} \mathrm{~s}^{-1}$ determined by Ibata et al. (2006). Because of our very large sample of member stars and our repeat measurements of individual stars to constrain our errors carefully, we conclude that there is no kinematically cold population in CVn I, and that there is no detectable difference between the kinematics and spatial distributions of the metal-rich and metalpoor stars.

Hercules.-The only galaxy in which we detect possible evidence of kinematic substructure is Hercules. There is a peak in the velocity histogram containing nine stars between 41 and $43 \mathrm{~km} \mathrm{~s}^{-1}$ (compared to the mean velocity of $45.0 \pm 1.1 \mathrm{~km} \mathrm{~s}^{-1}$ ), with the remaining 21 stars distributed more broadly between 30 and $60 \mathrm{~km} \mathrm{~s}^{-1}$. Given an intrinsically Gaussian distribution with the same mean velocity and velocity dispersion that we measure for Hercules, the likelihood of finding as many as nine stars out of 30 in such a narrow, offset peak is only $\sim 1 \%$. However, a twosided KS test indicates that the observed velocity distribution of stars in Hercules is consistent with a Gaussian at the $43 \%$ level. We conclude that there is not yet statistically significant evidence of velocity substructure in Hercules. If the velocity substructure does turn out to be real, it could be a sign in favor of the tidal disruption hypothesis advanced by Coleman et al. (2007).

Hercules contains two stars whose membership status is difficult to determine. The first of these, which is excluded from our member sample, has a velocity exactly on the mean velocity of the galaxy, but is offset almost $0.3 \mathrm{mag}$ from the RGB and is therefore rejected on the basis of the photometry. Because its velocity is so close to the mean of the other stars, this star would have a negligible effect on the measured velocity dispersion. The other candidate star, which does satisfy our membership criteria, has a velocity that is just within $3 \sigma$ of the mean velocity, at $30.7 \pm$ $2.2 \mathrm{~km} \mathrm{~s}^{-1}$. The Na equivalent width and metallicity of this star are also near the edge of the membership ranges; if we remove 
this star from the sample then the velocity dispersion of the galaxy would decrease to $4.2 \pm 0.9 \mathrm{~km} \mathrm{~s}^{-1}$.

\subsection{Tidal Disruption}

Two of the dwarfs presented in this paper show at least some evidence for ongoing tidal disruption by the Milky Way. As mentioned in $\S 3.5$, the properties of UMa II and perhaps Com appear to be affected by these interactions.

UMa II is located very close to the Milky Way, second only to Sagittarius (which is the archetype of tidally disrupting dwarfs) among the known dSphs. Zucker et al. (2006b) noted that UMa II appears irregular and that its stars are broken up into several subclumps. Belokurov et al. (2007a) pointed out that the Orphan Stream lies along a great circle intersecting the position of UMa II, and our measured radial velocity of $-116.5 \pm 1.9 \mathrm{~km} \mathrm{~s}^{-1}$ is in reasonable agreement with the $100 \mathrm{~km} \mathrm{~s}^{-1}$ predicted by Fellhauer et al. (2007) if UMa II is associated with the Orphan Stream. Fellhauer et al. also predict a roughly north-south velocity gradient over several degrees within UMa II. Although our member sample only spans a declination range of $13.6^{\prime}$, we do detect a modest correlation between radial velocity and declination among the member stars (correlation coefficient of -0.40 ), in the same sense as predicted. More significantly, we find strong evidence for a difference in the mean velocity between the eastern and western halves of the galaxy, with the stars on the eastern side having a velocity $8.4 \pm 1.4 \mathrm{~km} \mathrm{~s}^{-1}$ larger than those on the western side. It is highly unlikely that a galaxy as small as UMa II would show significant coherent rotation, so this velocity gradient strongly suggests that UMa II is distorted by tidal forces. As noted previously, UMa II is also a clear outlier from the $M_{V}-\sigma$ trend shown in Figure 10a. This galaxy therefore either has a mass-to-light ratio several times larger than any other dwarf (Table 4), or its velocity dispersion has been inflated by the tidal field of the Milky Way. Finally, UMa II has a metallicity $\gtrsim 0.5$ dex higher than would be expected from the luminosity-metallicity relationship shown in Figure 11. Its metallicity is more appropriate for a system with $M_{V} \approx-10$ (250 times more luminous than UMa II). Taken together, all of these independent results make a strong case for the imminent tidal disruption of UMa II, and we are not aware of any observational evidence suggesting that UMa II is bound.

Coma Berenices presents an intriguing counterpoint to UMa II. It shares some notable properties with UMa II, including an exceptionally low luminosity $\left(M_{V}=-3.7\right.$, compared to $M_{V}=$ -3.8), a location near the Milky Way (44 kpc instead of $32 \mathrm{kpc}$ ), and an unexpectedly high stellar metallicity. As with UMa II, we find a modest correlation of velocity with position in the galaxy (correlation coefficient of velocity with right ascension $=-0.24$ ). Dividing the galaxy in half along the minor axis, we find a mean velocity of $93.3 \pm 1.1 \mathrm{~km} \mathrm{~s}^{-1}$ for the northwestern side and a mean velocity of $98.8 \pm 0.5 \mathrm{~km} \mathrm{~s}^{-1}$ for the southeastern side. This velocity difference is significant at the $4 \sigma$ level. As with UMa II, it is not expected that galaxies of this size are rotationally supported, so if this velocity gradient is real it suggests that Coma Berenices, like UMa II, may be distorted by tidal forces. On the other hand, there are no known tidal streams that are plausibly associated with Com, its velocity dispersion is approximately what would be expected given its luminosity, and its stellar distribution is not noticeably more irregular than those of the other ultra-faint dwarfs (there are two bright stars immediately to the north of Com that may be responsible for the apparent distortion of the isopleths in that direction pointed out by Belokurov et al. 2007b). We also note that, with a smaller half-light radius (and larger central density; see $\S 4.3$ ) than any other Local Group dwarf, Com may be more robust to disruption than some of its counter- parts. While the available evidence is suggestive of the possibility that Coma Berenices could be tidally disrupting, the situation is not nearly as clear cut as it is for UMa II. We therefore treat Com as a bound, dark matter dominated object for now, while recognizing that future observations (most importantly, identification of an associated stellar stream) could change this picture.

For the other six galaxies in our sample, we do not detect any statistically significant velocity gradients or other evidence suggesting tidal disruption.

\section{DISCUSSION}

\subsection{The Missing Satellite Problem}

Understanding the nature of the ultra-faint dwarf galaxies and determining their impact on the missing satellite problem is one of the key goals of this work. Our observations show that with the likely exception of UMa II (and possibly Coma Berenices as well) the ultra-faint dwarfs seem to be dark matter dominated systems, with masses lower than those of the previously known $\mathrm{dSphs}$ and very large mass-to-light ratios. These galaxies are currently the darkest known stellar systems in the universe.

Determining the importance of the effect that the new dwarfs have on the abundance of satellite galaxies around the Milky Way requires having a way to compare observed galaxy properties to the properties of subhalos in $N$-body simulations. The simplest possible approach is to estimate the halo circular velocities of the ultra-faint dwarfs as $v_{\text {circ }}=\sqrt{3} \sigma$ (Klypin et al. 1999), assuming that the observed dispersions are equivalent to the maximum dispersions reached in each galaxy, and that the stars have negligible orbital anisotropy. Although these assumptions may not be correct in detail, if we use cumulative satellite distributions then the results of this exercise are relatively insensitive to them. The circular velocities of dark matter subhalos in the simulations can be measured robustly, giving us an appropriate point of comparison. We note that a more accurate means of comparing observed dwarfs to simulated subhalos is to use the mass contained within $0.6 \mathrm{kpc}$, which is better constrained by the observations than the halo circular velocity is (Strigari et al. 2007a); these calculations will be presented in a future paper (L. E. Strigari et al. 2008, in preparation). Using the above approximation, we find that the ultra-faint dwarfs have circular velocities from $v_{\text {circ }}=6-13 \mathrm{~km} \mathrm{~s}^{-1}$ (for plotting and comparison purposes, we round the circular velocity of Leo IV up to $6 \mathrm{~km} \mathrm{~s}^{-1}$ ). Because the fifth data release of the SDSS, where all of the new Milky Way satellites have been discovered, only covers $8000 \mathrm{deg}^{2}$ of sky, we must weight each of the new dwarfs by a factor of $\sim 5$ to account for the additional ultra-faint dwarfs likely to be discovered once the rest of the sky has been similarly surveyed.

We display the cumulative number of Milky Way satellites as a function of circular velocity in Figure 12. We assume Poisson uncertainties on the total number of dwarfs $\left[d N=\left(N_{\text {old }}+\right.\right.$ $\left.5^{2} N_{\text {new }}\right)^{1 / 2}$, where $N_{\text {old }}$ and $N_{\text {new }}$ refer to the previously known and newly discovered dwarf galaxies, respectively]. For comparison, we include the subhalo circular velocity function from the recent Via Lactea simulation, currently the highest resolution (234 million particles) $N$-body simulation of a Milky Way size galaxy (Diemand et al. 2007a, 2007b). This simulation assumes the best-fit $W M A P 3$ yr cosmological parameters: $\Omega_{m}=0.238, \Omega_{\Lambda}=$ $0.762, h=0.73, n=0.951$, and $\sigma_{8}=0.74$ (Spergel et al. 2007). The Via Lactea subhalos include all bound halos located within the virial radius $(389 \mathrm{kpc}$ ) of the main halo (see also $\S 4.1 .2)$. The addition of the new dwarfs, combined with the correction for the sky area that has yet to be observed with sufficient sensitivity, substantially changes the appearance of the substructure problem. The 


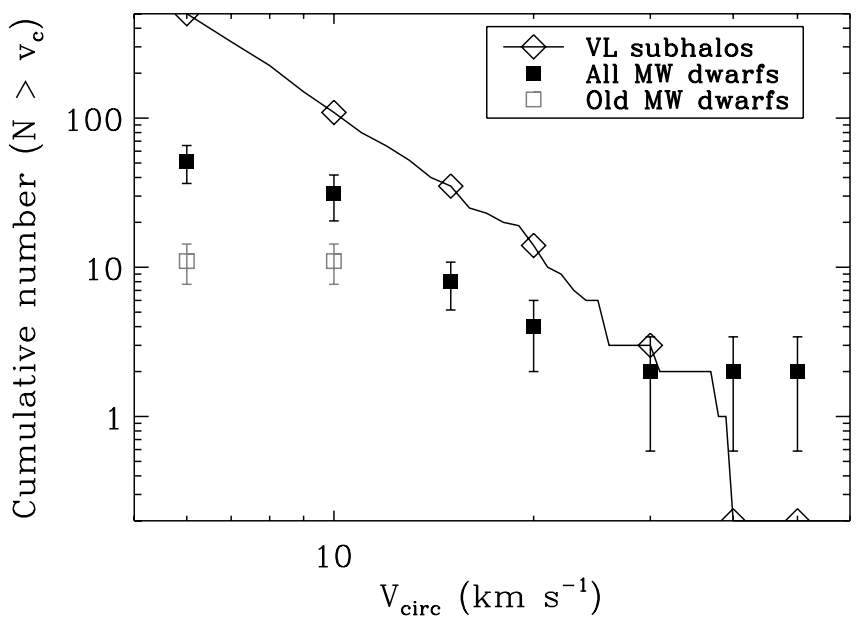

FIG. 12.-Cumulative number of Milky Way satellite galaxies as a function of halo circular velocity. The filled black squares include the new circular velocity estimates from this paper (plus Boötes, but excluding UMa II), as well as all of the previously known Milky Way dwarfs. The open gray squares show the observed distribution without the new ultra-faint dwarfs. We assume Poisson errors on the number count of satellites in each bin (computed independently for the new and old dwarfs), although the true uncertainties may be larger. The solid line with diamonds represents the subhalo abundance within the virial radius of the Via Lactea $N$-body simulation (Diemand et al. 2007a).

previously known Milky Way satellite galaxies have a nearly flat circular velocity function below $v_{\text {circ }}=15 \mathrm{~km} \mathrm{~s}^{-1}$, causing a discrepancy with the predictions that worsens with decreasing mass and reaches well over an order of magnitude below $v_{\text {circ }}=10 \mathrm{~km} \mathrm{~s}^{-1}$. With the ultra-faint dwarfs included we now see a rising circular velocity function and a satellite underabundance of a factor of $\sim 4$ for halos with masses between $v_{\text {circ }}=10$ and $20 \mathrm{~km} \mathrm{~s}^{-1}$. At $v_{\text {circ }}=6 \mathrm{~km} \mathrm{~s}^{-1}$ the discrepancy increases again toward an order of magnitude, but if the current observational census is still incomplete at the faint end, this is the mass range where that would manifest itself. The ultra-faint dwarfs significantly fill in the gap for satellites in the two lowest mass bins, but have masses that are too small to affect the satellite deficit at higher circular velocities.

\subsubsection{Proposed Solutions to the Missing Satellite Problem}

Using these new data, we can test a number of proposed astrophysical solutions to the missing satellite problem. For example, the observed dwarf galaxies could inhabit the most massive subhalos at the present day (Stoehr et al. 2002), the subhalos that collapsed at the highest redshift (Bullock et al. 2000), or the subhalos that were the most massive at the time they were accreted by the Milky Way (Kravtsov et al. 2004). We show the results of these tests in Figures 13 and 14. To compare the observed dwarfs to the most massive (MM) subhalos, we identified the 51 halos (to match the number of Milky Way satellites projected to be found once the remainder of the sky has been surveyed) located within the virial radius that have the largest total masses at the present day in the Via Lactea simulation. The circular velocity function of these subhalos is plotted as the solid cyan curve in Figure 13. Note that because we chose the total number of subhalos to match the total number of Milky Way dwarfs, the agreement between the observed distribution and the cyan curve in the lowest mass bin is trivial. Another possibility is to compare the observed circular velocity function with the circular velocity function of the subhalos that were most massive when they were accreted (Fig. 13, dashed purple curve). We selected the largest before accretion (LBA) subhalos from the Via Lactea simulation as the halos located within

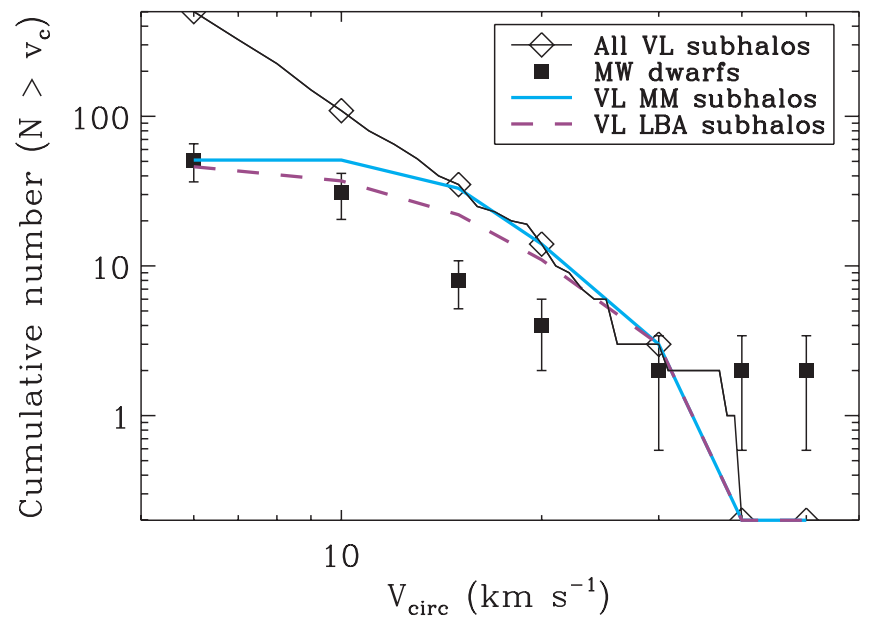

FIG. 13.- Outcome of two proposed solutions to the missing satellite problem. As in Fig. 12, the filled black squares include the new circular velocity estimates from this paper ( plus Boötes, but excluding UMa II), as well as all of the previously known Milky Way dwarfs, and the solid line with diamonds represents the subhalo abundance within the virial radius of the Via Lactea $N$-body simulation (Diemand et al. 2007a). The solid cyan curve shows the circular velocity distribution for the 51 most massive Via Lactea subhalos at $z=0$. The dashed purple curve illustrates the circular velocity distribution for the $51 \mathrm{Via}$ Lactea subhalos that had the largest masses at the time they were accreted by the main halo.

the virial radius of the main halo at $z=0$ that had the largest circular velocities at any point in the past. Again, the agreement at the low-mass end is simply a result of our choice of the top 51 subhalos from the simulation. ${ }^{6}$ If the observed dwarf galaxies inhabit only the most massive dark matter subhalos around the Milky Way, the shape of the mass function of the most massive subhalos fails to match the shape of the observed mass function. Using the subhalos that were most massive at the time they were accreted

6 The largest before accretion subsample at the present day (Fig. 13, dashed purple curve) actually only has 46 objects with $v_{\text {circ }}>6 \mathrm{~km} \mathrm{~s}^{-1}$ because five of the subhalos lost so much mass by $z=0$ that they ended up with even lower presentday circular velocities than are shown in the plot.

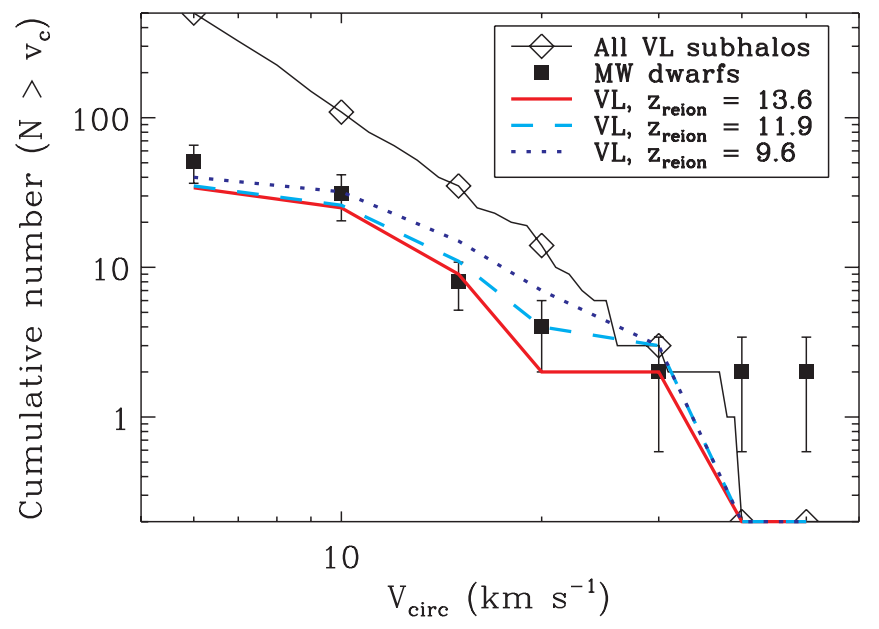

FIG. 14.-Effect of reionization on the missing satellite problem. As in Fig. 12, the filled black squares include the new circular velocity estimates from this paper (plus Boötes, but excluding UMa II), as well as all of the previously known Milky Way dwarfs, and the solid line with diamonds represents the subhalo abundance within the virial radius of the Via Lactea $N$-body simulation (Diemand et al. 2007a). The solid red curve shows the circular velocity distribution for the 51 most massive Via Lactea subhalos at $z=13.6$, the dashed cyan curve at $z=11.9$, and the dotted blue curve at $z=9.6$. 
instead of the ones most massive today (i.e., allowing for mass lost by tidal stripping) brings the subhalo mass function slightly closer to the observed one, but there are still a factor of $\sim 3$ too few dwarfs in the $v_{\text {circ }}=10-30 \mathrm{~km} \mathrm{~s}^{-1}$ range.

The final astrophysical solution we consider is that only halos that collapsed prior to reionization were able to form significant numbers of stars (e.g., Bullock et al. 2000; Somerville 2002; Moore et al. 2006). Among the Via Lactea subhalos that are located within the virial radius at $z=0$, we select the objects with the 51 largest values of $v_{\text {circ }}$ at various high redshifts. The results of this test are displayed in Figure 14. The solid red, dashed cyan, and dotted blue curves represent the subhalos that would be selected if $z_{\text {reion }}=13.6,11.9$, and 9.6, respectively. If reionization occurred around redshift 9-14, and dwarf galaxy formation was strongly suppressed thereafter, the circular velocity function of Milky Way satellite galaxies approximately matches that of CDM subhalos. If reionization occurred at $z \lesssim 8$, we again find an underabundance of Milky Way dwarfs with $v_{\text {circ }}=15-30 \mathrm{~km} \mathrm{~s}^{-1}$ compared to theoretical models, although we note that the individual subhalo $v_{\text {circ }}(z)$ histories in the Via Lactea simulation are noisy at high redshift, and the number of objects in these bins is relatively low. We therefore suggest that the observed mass function of Milky Way satellite galaxies constrains reionization to have taken place before $z=8$, in agreement with the $3 \mathrm{yr} W M A P$ results from measurements of the cosmic microwave background $\left(z_{\text {reion }}=\right.$ $10.9_{-2.3}^{+2.7}$; Page et al. 2007). However, there are a number of caveats to this analysis: (1) the extrapolation of dwarf galaxy abundances from the SDSS DR5 sky coverage to the whole sky must be reasonable; (2) the observed velocity dispersions must provide a reasonable estimate of the halo circular velocities; (3) the primary physical mechanism responsible for suppressing the formation of galaxies in low-mass dark matter halos must be reionization; (4) the cosmology used for the Via Lactea simulation (Diemand et al. 2007a, 2007b) — particularly the low value of $\sigma_{8}$ — must be a good match to the cosmology of our universe; and (5) the main halo simulated in Via Lactea must be a reasonable representation of the Milky Way. We also note that while WMAP and most other observational probes are sensitive to the mean reionization history of the universe, the dwarf galaxies observed in the study are sensitive primarily to the reionization history of the Local Group. If reionization was indeed responsible for the low abundance of Galactic satellites, then the Milky Way and/or M31 must have been undergoing vigorous enough star formation to ionize the intergalactic medium of the Local Group at $z>8$.

\subsubsection{Observational Incompleteness and the Comparison Radius in Simulations}

One of the important assumptions involved in our analysis in $\S \S 4.1$ and 4.1 .1 is the choice of the radius in the simulations out to which satellites should be counted. In the ideal case, this radius should be the virial radius, as we have used, but in reality the comparison between observations and simulations is only meaningful in the regime where the observations are complete.

The observational census for Milky Way satellite galaxies similar to the brighter dwarf spheroidals $\left(M_{V} \lesssim-9\right)$ should be largely complete by now; the last Milky Way satellite to be discovered in this luminosity range was Sagittarius (Ibata et al. 1994). Recent searches of Palomar Sky Survey data, which are sensitive to such galaxies anywhere within the Local Group, have not detected additional dwarfs (Whiting et al. 2007; Simon \& Blitz 2002). The distribution of Milky Way dwarf galaxies as a function of Galactic latitude suggests that additional relatively bright dwarfs remain to be discovered at low latitudes, where extinction and foreground confusion are serious problems. The expected number of such ob- jects if they are distributed uniformly around the Galaxy is $\sim 4$ (Mateo 1998; Willman et al. 2004), which does not appear to be enough to significantly affect the missing satellite problem.

Very recently, Koposov et al. (2007) have analyzed the detectability of faint Milky Way satellites in the SDSS DR5 data. They find that extremely low-luminosity objects $\left(M_{V} \lesssim-5\right)$ may be missed by SDSS searches if they are located at relatively large distances $(d \gtrsim 100 \mathrm{kpc})$, as the horizontal branch and MSTO stars that their detection relies on become too faint to be reliably detected in the SDSS. Galaxies with even lower surface brightnesses than the known dwarfs $\left(\mu_{V} \gtrsim 30\right.$ mag $\left.\operatorname{arcsec}{ }^{-2}\right)$, if they exist, are also likely to have escaped detection. However, if there is a correlation between surface brightness and distance from the host galaxy (e.g., McConnachie \& Irwin 2006) or density and distance (as our data and Mayer et al. [2001b] suggest), there may not be significant numbers of ultra-faint dwarfs at large distances. For more luminous dwarfs and those with higher central surface brightnesses, the current sample of Milky Way satellites should be reasonably complete. The Koposov et al. (2007) luminosity function of Milky Way satellite galaxies predicts that there are a total of 57 dwarf galaxies within $280 \mathrm{kpc}$ of the Milky Way over the whole sky, and a similar number within $420 \mathrm{kpc}$. Thus, our much simpler estimates of $46 \pm 14$ dwarf galaxies within $250 \mathrm{kpc}$ and $51 \pm 15$ within $420 \mathrm{kpc}$ from $\S 4.1$ appear to be well justified.

Incompleteness may still be a significant problem at the extreme faint end of the luminosity function, as the recent discovery of Boötes II reveals (Walsh et al. 2007). Our results suggest that satellites in this luminosity range are not gravitationally bound dwarf galaxies. If these objects are tidally disrupted dwarfs, then they should still contribute to the Milky Way satellite census, but if they are simply multiple fragments from larger objects (for example, if there is a physical connection between Boötes and Boötes II) or unusual globular clusters then they do not correspond to dark matter subhalos in the CDM simulations. Until surveys are more complete at faint magnitudes and some kinematic information is available for this class of objects, their effect on the missing satellite problem is not clear.

In case there are undiscovered ultra-faint Milky Way dwarfs beyond $d=250 \mathrm{kpc}$, we repeated our analysis of $\S 4.1 \mathrm{using}$ subhalos within $250 \mathrm{kpc}$ from the main halo in the Via Lactea simulation (and discarding Leo $\mathrm{T}$ from the observed sample because it is beyond this radius). This smaller radius reduces the overall number of satellite subhalos by $\sim 30 \%$, not enough to significantly change our conclusions. Limiting the comparison to this radius shifts the preferred range of reionization redshifts slightly lower, but also lessens our leverage on determining the redshift of reionization.

\subsection{The Constant Halo Mass Hypothesis}

Mateo et al. (1993) was the first to point out that observations of the dSphs known at that time suggested that they were all embedded within dark matter halos of mass $\sim 3 \times 10^{7} M_{\odot}$, independent of luminosity. In Figure 15 we display an updated version of what has become popularly known as the "Mateo" plot, showing the mass-to-light ratios of all of the Local Group dSphs with measured kinematics as a function of absolute magnitude. As seen previously by Mateo et al. (1993), Mateo (1998), and Gilmore et al. (2007), all of the galaxies observed prior to this work are approximately consistent with the picture proposed by Mateo et al. (1993). The results change, however, when the ultrafaint Milky Way satellites are added. Although the brightest of the ultra-faint dwarfs still lie within the same range of halo mass as their more luminous counterparts, the fainter objects (Hercules, Leo IV, CVn II, and Coma Berenices) are located well below 


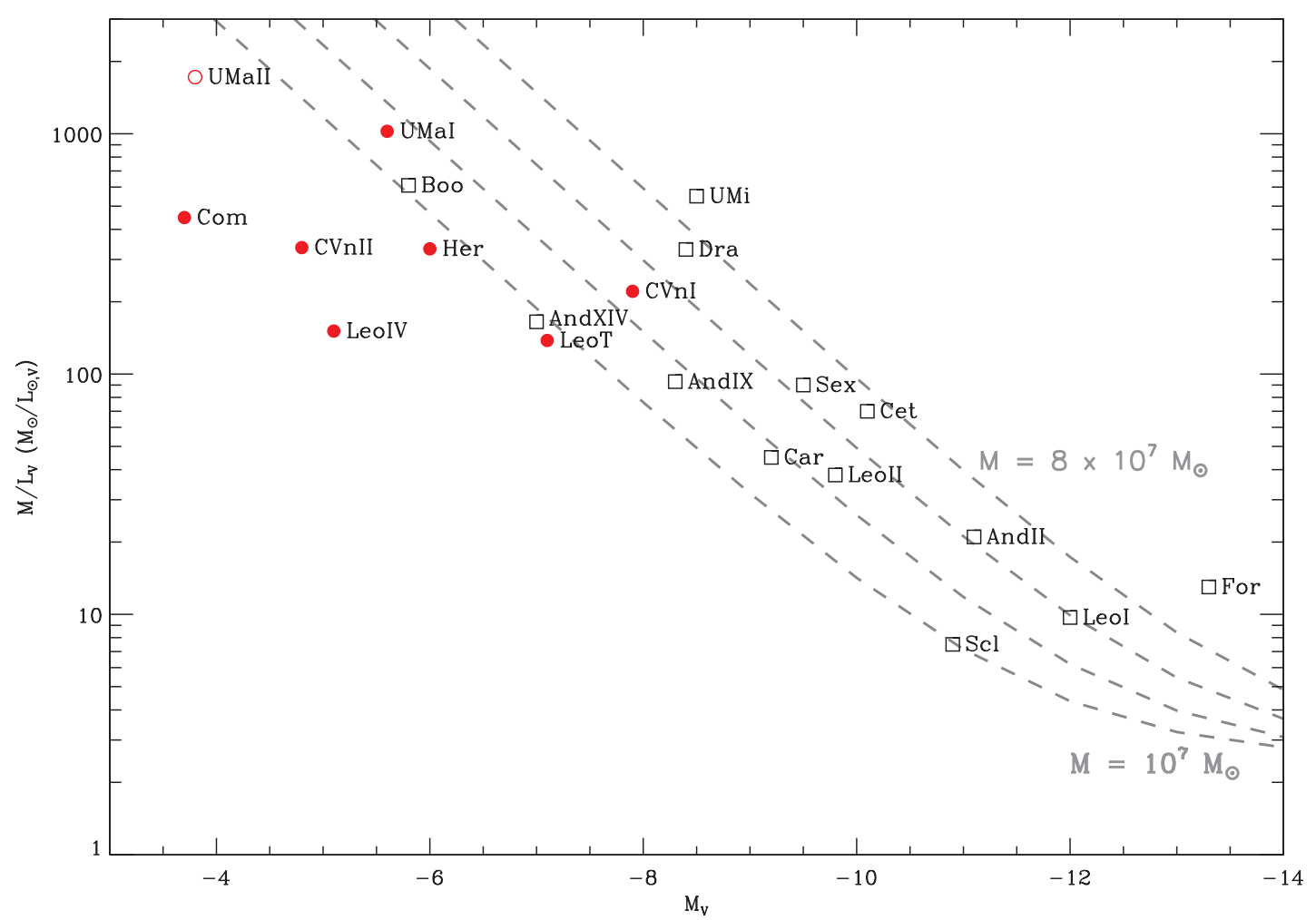

FIG. 15. - Total mass-to-light ratios (in solar units) as a function of absolute magnitude for Local Group dwarf spheroidals. The red symbols represent the ultra-faint dwarfs from this paper (including Leo T, which is not really a dSph, and UMa II, which may be tidally disrupted, as an open red circle at the upper left). The open black squares represent all of the dSphs with previously published kinematic data, including satellites of M31 as well as the Milky Way. The dashed gray lines are curves of constant dark matter halo mass $\left(1,2,4\right.$, and $8 \times 10^{7} M_{\odot}$, from bottom to top), assuming a stellar mass-to-light ratio of $2.5 M_{\odot} / L_{\odot, V}$. For the previously known Milky Way dwarfs, we recomputed luminosities from Irwin \& Hatzidimitriou (1995) using the most up-to-date distance measurements, and then adjusted the mass-to-light ratios from the literature accordingly. References for distance measurements are Fornax: Saviane et al. (2000), Mackey \& Gilmore (2003), Gullieuszik et al. (2007); Leo I: Bellazzini et al. (2004); Sculptor: Mateo (1998); Leo II: Bellazzini et al. (2005); Sextans: Lee et al. (2003); Carina: Dall'Ora et al. (2003); Ursa Minor: Mighell \& Burke (1999); and Draco: Bonanos et al. (2004). References for $M / L$ measurements are Fornax: Walker et al. (2006a); Leo I: Sohn et al. (2007), Koch et al. (2007b); Sculptor: Westfall et al. (2006); Leo II: Koch et al. (2007a); Sextans: Walker et al. (2006b); Carina: Muñoz et al. (2006b); Ursa Minor: Wu (2007); Draco: Łokas et al. (2005); And II: Côté et al. (1999); Cetus: Lewis et al. (2007); And IX: Chapman et al. (2005); And XIV: Majewski et al. (2007); and Boötes: Muñoz et al. (2006a).

the extrapolated trend. These galaxies have much lower halo masses, and hence their mass-to-light ratios are significantly smaller than what would be expected if they too were embedded in $\sim 3 \times 10^{7} M_{\odot}$ halos. Combining the new and old dwarfs, it appears that there are two distinct regimes: the brighter dwarfs $\left(M_{V}<-9\right)$ all have similar-mass dark matter halos, but for the fainter dwarfs $\left(M_{V}>-9\right) M / L$ saturates at a value of $200-1000$ and the halo mass declines as luminosity decreases (see Fig. 10b). It therefore appears that the ultra-faint dwarfs do not occupy halos as massive as those of the "normal" dSphs; if there is a minimum halo mass for dwarf galaxies, it is not clear that the observations have yet reached it.

\subsection{Central Dark Matter Densities in the Ultra-faint Dwarfs}

The observed velocity dispersions and radii of the new dwarfs constrain their densities as well as their masses and mass-to-light ratios. Because these galaxies are highly dark matter dominated, the overall densities we derive are essentially equal to the density of the dark matter halo of each object. Following Mateo et al. (1991), we can approximate the central density as

$$
166 \sigma^{2} \eta / r_{c}^{2},
$$

where $\eta$ is a numerical parameter that works out to 1 for plausible density profiles. The central densities of the new dwarfs range from $\sim 0.08 M_{\odot} \mathrm{pc}^{-3}$ for CVn I and Hercules up to $\sim 2.1 M_{\odot} \mathrm{pc}^{-3}$ for the faintest galaxy, Coma Berenices. Alternatively, we can cal- culate mean densities from the total masses given in Table 4 and the core radii. To compare with the mean densities of the previously known Milky Way dSphs tabulated by Gilmore et al. (2007), we assume that the extent of the new dwarfs is $\sim 2$ King core radii. Again, Coma Berenices is the densest object, with a mean density of $0.52 M_{\odot} \mathrm{pc}^{-3}\left(=20 \mathrm{GeV} / \mathrm{c}^{2} \mathrm{~cm}^{-3}\right)$, almost a factor of 5 higher than any of the previously known $\mathrm{dSphs}$. This substantially raises the limiting mass density of $\sim 5 \mathrm{GeV} / \mathrm{c}^{2} \mathrm{~cm}^{-3}$ identified by Gilmore et al. (2007) and suggests that there may not be a true physical ceiling on the densities of dwarf galaxies (as opposed to an observational ceiling) at all. However, if Com is in the process of tidal disruption, as our observations hint, then the highest dark matter densities in our sample occur in Leo T and CVn II and are only modestly above the density limit of Gilmore et al. (2007).

\subsection{Phase-Space Density Constraints from the Ultra-faint Dwarfs}

Hogan \& Dalcanton (2000) introduced the parameter $Q \equiv \rho / \sigma^{3}$ as an estimate of the coarse-grained phase-space density of the dark matter in galaxy halos. As discussed by Hogan \& Dalcanton (2000), Dalcanton \& Hogan (2001), and Strigari et al. (2006), Liouville's theorem implies that observed values of $Q$ set a hard lower limit on the original phase-space density of the dark matter. By finding the systems with the largest observed values of $Q$, we can therefore constrain the properties of dark matter and potentially rule out classes of dark matter candidates. Observations of low-mass spiral galaxies by Simon et al. (2005) yield lower 
TABLE 5

Physical and Phase-Space Densities

\begin{tabular}{cccc}
\hline \hline Galaxy & $\begin{array}{c}\rho_{0} \\
\left(M_{\odot} \mathrm{pc}^{-3}\right)\end{array}$ & $\begin{array}{c}\bar{\rho} \\
\left(M_{\odot} \mathrm{pc}^{-3}\right)\end{array}$ & $\begin{array}{c}Q \\
{\left[M_{\odot} \mathrm{pc}^{-3}\left(\mathrm{~km} \mathrm{~s}^{-1}\right)^{-3}\right]}\end{array}$ \\
\hline Ursa Major II ${ }^{\mathrm{a}} \ldots \ldots \ldots .$. & $1.13 \pm 0.60$ & $0.27 \pm 0.18$ & $(3.7 \pm 3.1) \times 10^{-3}$ \\
Leo T................... & $0.79 \pm 0.36$ & $0.19 \pm 0.10$ & $(1.9 \pm 1.5) \times 10^{-3}$ \\
Ursa Major I ............ & $0.25 \pm 0.08$ & $0.06 \pm 0.02$ & $(5.6 \pm 2.9) \times 10^{-4}$ \\
Leo IV ................... & $0.19 \pm 0.20$ & $0.05 \pm 0.05$ & $(5.3 \pm 9.9) \times 10^{-3}$ \\
Coma Berenices ........ & $2.09 \pm 0.86$ & $0.52 \pm 0.24$ & $(2.2 \pm 1.4) \times 10^{-2}$ \\
Canes Venatici II........ & $0.49 \pm 0.25$ & $0.12 \pm 0.07$ & $(5.1 \pm 4.1) \times 10^{-3}$ \\
Canes Venatici I......... & $0.08 \pm 0.02$ & $0.02 \pm 0.01$ & $(1.7 \pm 0.5) \times 10^{-4}$ \\
Hercules ..................... & $0.10 \pm 0.04$ & $0.02 \pm 0.01$ & $(7.7 \pm 5.2) \times 10^{-4}$ \\
\hline
\end{tabular}

${ }^{\text {a }}$ UMa II may be a tidally disrupted remnant, which would artificially inflate its density.

limits of $\sim 10^{-6} M_{\odot} \mathrm{pc}^{-3}\left(\mathrm{~km} \mathrm{~s}^{-1}\right)^{-3}$ on $Q$ (Strigari et al. 2006; G. Martinez et al. 2008, in preparation), but those are less restrictive constraints than are provided by the Ly $\alpha$ forest. $Q$ values for the ultra-faint dwarfs are listed in Table 5. These values are calculated under the assumption that the velocity dispersion of the dark matter (which is what $Q$ actually depends on) is equal to the velocity dispersion of the stars. Our observations show that most of the ultra-faint dwarfs greatly exceed the phase-space density constraint from the Ly $\alpha$ forest, reaching a maximum of $Q=2$. $2 \times 10^{-2} M_{\odot} \mathrm{pc}^{-3}\left(\mathrm{~km} \mathrm{~s}^{-1}\right)^{-3}$ in Coma Berenices. Even if the derived $Q$ of Com has been affected by tidal disruption, all of the galaxies except UMa I, CVn I, and Hercules have $Q$ values $\gtrsim 10^{-3} M_{\odot} \mathrm{pc}^{-3}\left(\mathrm{~km} \mathrm{~s}^{-1}\right)^{-3}, 2$ orders of magnitude better than the Ly $\alpha$ forest constraint and about an order of magnitude improvement compared to the previously known dSphs. In fact, the dark matter velocity dispersion is expected to be larger than the stellar velocity dispersion, so the $Q$ values we derive are upper limits on the true $Q$ values for these galaxies. Nevertheless, these $Q$ values will further restrict the allowed parameter space for warm dark matter particles, and may have an impact on the metaCDM scenario proposed by Strigari et al. (2007b).

\section{SUMMARY AND CONCLUSIONS}

We have obtained Keck DEIMOS spectra of significant samples of stars in eight of the new, ultra-faint Milky Way satellite galaxies recently discovered in the Sloan Digital Sky Survey. Using a large spectroscopic data set of radial velocity standard stars observed with DEIMOS, repeat DEIMOS measurements of stars in dwarf spheroidals and globular clusters, and DEIMOS and HIRES spectra of the same stars, we demonstrated that both our velocity measurements and our derived uncertainties are accurate. We then measured the velocities of 18-214 stars in each galaxy, with typical uncertainties of $\sim 3.4 \mathrm{~km} \mathrm{~s}^{-1}$.

From our measurements of individual stellar velocities, we calculated velocity dispersions for each of the ultra-faint dwarfs. The velocity dispersions, which are listed in Table 3 , range from $3.3 \pm 1.7 \mathrm{~km} \mathrm{~s}^{-1}$ for Leo IV up to $7.6 \pm 0.4 \mathrm{~km} \mathrm{~s}^{-1}$ for CVn I, and we showed that the velocity dispersions are correlated with luminosity (inversely correlated with absolute magnitude). Under a set of simple assumptions, we calculated the total masses of the ultra-faint dwarfs, finding that these objects are the lowestmass galaxies currently known. From the equivalent widths of the Ca triplet absorption lines we measured the metallicities of the RGB stars in the new dwarfs and derived mean metallicities ranging from $[\mathrm{Fe} / \mathrm{H}]=-2.0$ to $[\mathrm{Fe} / \mathrm{H}]=-2.3$; several of these galaxies are the most metal-poor stellar systems yet discovered.
We summarize our primary conclusions from this study as follows:

1. The ultra-faint Milky Way satellites are dark matter dominated dwarf galaxies with lower masses than any other known galaxies.

2. The only clear exception among the eight galaxies we observed, as well as those previously observed by others, is Ursa Major II. Based on its clumpy appearance (Zucker et al. 2006b), small galactocentric distance (Zucker et al. 2006b), associated tidal stream (Fellhauer et al. 2007) (§ 3.5), inflated velocity dispersion ( $\S 3.2)$, unusually high metallicity ( $\S 3.4)$, and possible velocity gradient $(\S 3.5)$, we conclude that UMa II is in the late stages of tidal disruption. The other dwarf with $M_{V} \gtrsim-4$, Coma Berenices, has a similarly high metallicity that indicates it may have suffered substantial tidal stripping as well. Because Com lacks most of the other supporting evidence for tidal disruption, we assume for now that it is still a bound, dark matter dominated dwarf, although we recognize that future observations may show otherwise. Based on these results, we suggest that $M_{V} \approx-4(3.4 \times$ $\left.10^{3} L_{\odot}\right)$ is the lower limit to the luminosity of gravitationally bound dwarf galaxies. We therefore predict that objects such as Willman 1, Segue 1, and Boötes II will prove to be tidally disrupted remnants.

3. The six ultra-faint dwarfs with $M_{V} \lesssim-4$ follow the luminosity-metallicity relationship established by the more luminous Local Group dwarfs, and extend the relation by $\sim 2$ orders of magnitude in luminosity. The faintest dwarfs, UMa II and Com, are both outliers from this relationship, with metallicities more than 0.5 dex too large for their luminosities (or conversely, luminosities that are more than 2 orders of magnitude too small for their metallicities). We detect metallicity spreads of up to 0.5 dex in several objects, suggesting multiple star formation epochs.

4. The total mass-to-light ratios of the ultra-faint dwarfs reach as high as $1000 M_{\odot} / L_{\odot, V}$ (UMa I). While the brighter galaxies $\left(M_{V} \lesssim-9\right)$ have mass-to-light ratios consistent with the hypothesis that all dwarf spheroidals are embedded within dark matter halos of the same mass, the fainter galaxies depart from this trend in the sense that their mass-to-light ratios are too low (i.e., they have lower masses). We therefore suggest that the minimum mass for dwarf galaxies (as opposed to the minimum luminosity mentioned earlier), if there is one, may not have been reached yet.

5. The ultra-faint Milky Way satellites, after correcting for the sky area not covered by DR5 of the Sloan survey, substantially increase the abundance of dwarf galaxies with very low masses $\left(v_{\text {circ }} \leq 15 \mathrm{~km} \mathrm{~s}^{-1}\right)$, thereby reducing the satellite deficit compared to CDM simulations to a factor of $\sim 4$. Proposals to remedy the missing satellite problem by placing the observed dwarf galaxies in the most massive dark matter subhalos (at the present day) around the Milky Way or in the subhalos that were most massive at the time they were accreted by the Milky Way do not reproduce the observed shape of the circular velocity function. If we assume instead that only the halos that acquired a significant amount of mass ( $v_{\text {circ }} \gtrsim 8 \mathrm{~km} \mathrm{~s}^{-1}$, varying somewhat with $z_{\text {reion }}$ ) before the redshift of reionization were able to form stars, then the subhalos from the Via Lactea simulation (Diemand et al. 2007a, 2007b) approximately match both the total number of Milky Way dwarfs and the shape of the circular velocity function.

6. The central dark matter densities of the ultra-faint dwarfs are as high as $2.1 M_{\odot} \mathrm{pc}^{-3}\left(0.8 M_{\odot} \mathrm{pc}^{-3}\right.$ if Coma Berenices is tidally disrupting), significantly larger than those of the previously known dwarf spheroidals. The phase-space densities are also higher than those of other astrophysical systems $\left[Q>10^{-3} M_{\odot} \mathrm{pc}^{-3}\right.$ $\left.\left(\mathrm{km} \mathrm{s}^{-1}\right)^{-3}\right]$, which will place significant limits on non-CDM dark matter models. 
Data presented herein were obtained at the W. M. Keck Observatory, which is operated as a scientific partnership among the California Institute of Technology, the University of California, and the National Aeronautics and Space Administration. The observatory was made possible by the generous financial support of the W. M. Keck Foundation. The authors wish to recognize and acknowledge the very significant cultural role and reverence that the summit of Mauna Kea has always had within the indigenous Hawaiian community. We are most fortunate to have the opportunity to conduct observations from this mountain. J. D. S. gratefully acknowledges the support of a Millikan Fellowship provided by Caltech and M. G. acknowledges support from a Plaskett Research Fellowship at the Herzberg Institute of Astrophysics of the National Research Council of Canada. We thank George Djorgovski, Vasily Belokurov, Leo Blitz, James Bullock, Judy Cohen, Pat Côté, Jürg Diemand, Gerry Gilmore, Raja
Guhathakurta, Nicolas Martin, Emma Ryan-Weber, Wal Sargent, Peter Stetson, Louie Strigari, Beth Willman, and Dan Zucker for helpful conversations, and we acknowledge the useful suggestions of the anonymous referee. We also thank Michael Cooper and the DEEP2 team for their hard work on the DEIMOS data reduction pipeline. The analysis pipeline used to reduce the DEIMOS data was developed at UC Berkeley with support from NSF grant AST-0071048. This research has made use of NASA's Astrophysics Data System Bibliographic Services, the NASA/IPAC Extragalactic Database (NED), which is operated by the Jet Propulsion Laboratory, California Institute of Technology, under contract with the National Aeronautics and Space Administration, and the SIMBAD database, operated at CDS, Strasbourg, France.

Facilities: Keck:II (DEIMOS)

\section{APPENDIX}

\section{COLLECTING UNIFORM DATA FOR THE ULTRA-FAINT DWARF GALAXIES}

The new ultra-faint dwarf galaxies have been discovered by a number of different authors, which means that their properties have not all been determined in a uniform manner. In order to calculate masses and mass-to-light ratios as consistently as possible for each galaxy, and to facilitate future studies of these objects, in this Appendix we collect the currently available data on all 12 of the new Milky Way satellites (see Table 6).

With the exception of Willman 1 and Boötes II, we use the absolute magnitudes and radii determined by the Cambridge group (references given in Tables 1 and 6) from the SDSS data. This includes the revised absolute magnitude of $M_{V}=-5.5$ for UMa I reported in Belokurov et al. (2006), which differs substantially from the original value of $M_{V}=-6.75$ estimated by Willman et al. (2005a), although the uncertainties on both numbers are admittedly large. Note that our improved distance for UMa I of $106 \mathrm{kpc}$ (compared to the previously reported $100 \mathrm{kpc}$ ) requires a corresponding change in the absolute magnitude to $M_{V}=-5.6$. The Belokurov et al. magnitude for UMa I is not accompanied by an uncertainty; by analogy to the other galaxies of similar luminosity we assume an uncertainty of 0.6 mag. Belokurov et al. have also remeasured the radius of UMa I in the same manner as they did for the other Milky Way galaxies, finding a Plummer radius of $10^{\prime}$ (D. Zucker \& V. Belokurov 2007, private communication).

UMa II is described in the discovery paper only as having an angular extent of $\sim 0.5^{\circ} \times 0.25^{\circ}$ and a half-light radius of approximately $120 \mathrm{pc}$ (Zucker et al. 2006b). Similarly to UMa I, the authors have refit the light profile using the same method as they did for the other new dwarfs and measured a half-light radius of 13.6' (D. Zucker \& V. Belokurov 2007, private communication). Note that unlike the Plummer radii we use for the other dwarfs, this radius is the mean of the Plummer radius and the exponential scale radius; in most cases the two radii are very similar.

Boötes II does not have a published distance uncertainty, so given the angular proximity to Boötes and the apparently identical distance moduli, we assume the same distance uncertainty for Boötes II as Belokurov et al. (2006) derived for Boötes.

Most of the new discoveries do not have published uncertainties for their Plummer radius fits. For these objects, we assume an uncertainty of $6 \%$ (the uncertainty given for $\mathrm{CVn} \mathrm{I}$ ) on the angular radius for the purposes of calculating the uncertainty on the corresponding physical radius.

TABLE 6

Parameters of the Ultra-faint Milky Way Satellites

\begin{tabular}{|c|c|c|c|c|c|}
\hline Galaxy & $M_{V}$ & $\begin{array}{c}\text { Distance } \\
(\mathrm{kpc})\end{array}$ & $\begin{array}{l}r_{\text {Plummer }} \\
\text { (arcmin) }\end{array}$ & $\begin{array}{l}r_{\text {Plummer }} \\
(\mathrm{pc})\end{array}$ & References \\
\hline Ursa Major II ............................ & $-3.8 \pm 0.6$ & $32_{-4}^{+5}$ & 13.6 & $127 \pm 21$ & 1,2 \\
\hline Leo T..................................... & $-7.1 \pm 0.3$ & $417^{+20}$ & 1.4 & $170 \pm 15$ & 3 \\
\hline Ursa Major I ........................... & $-5.6 \pm 0.6$ & $106_{-8}^{+19}$ & 10.0 & $308 \pm 32$ & $2,4,5$ \\
\hline 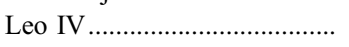 & $-5.1 \pm 0.6$ & $158_{-14}^{+15}$ & 3.3 & $152 \pm 17$ & 6 \\
\hline Coma Berenices ........................ & $-3.7 \pm 0.6$ & $44 \pm 4$ & 5.0 & $64 \pm 7$ & 6 \\
\hline Canes Venatici II...................... & $-4.8 \pm 0.6$ & $151_{-13}^{+15}$ & 3.0 & $132 \pm 16$ & 6 \\
\hline Canes Venatici I..................... & $-7.9 \pm 0.5$ & $224_{-20}^{+22}$ & $8.5 \pm 0.5$ & $554 \pm 63$ & 7 \\
\hline 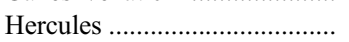 & $-6.0 \pm 0.6$ & $138_{-12}^{+13}$ & 8.0 & $321 \pm 36$ & 6 \\
\hline 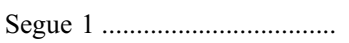 & $-3.0 \pm 0.6$ & $23 \pm 2$ & 4.5 & $30 \pm 3$ & 6 \\
\hline 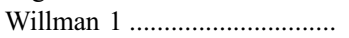 & $-2.5 \pm 1.0$ & $38 \pm 7$ & $1.9 \pm 0.3$ & $21 \pm 5$ & 8 \\
\hline (n..................... & $-3.1 \pm 1.1$ & $60 \pm 6$ & $4.1 \pm 1.6$ & $72 \pm 28$ & 9 \\
\hline 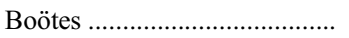 & $-5.8 \pm 0.5$ & $60 \pm 6$ & $13.0 \pm 0.7$ & $227 \pm 26$ & 3 \\
\hline
\end{tabular}

REFERENCES.-(1) Zucker et al. 2006b; (2) D. Zucker \& V. Belokurov 2007, private communication; (3) Irwin et al. 2007; (4) Belokurov et al. 2006; (5) this work; (6) Belokurov et al. 2007b; (7) Zucker et al. 2006a; (8) Willman et al. 2006; (9) Walsh et al. 2007. 
Adelman-McCarthy, J., et al. 2007, ApJS, 172, 634

Bellazzini, M., Gennari, N., \& Ferraro, F. R. 2005, MNRAS, 360, 185

Bellazzini, M., Gennari, N., Ferraro, F. R., \& Sollima, A. 2004, MNRAS, 354, 708

Belokurov, V., et al. 2006, ApJ, 647, L111 2007a, ApJ, 658, 337 2007b, ApJ, 654, 897

Benson, A. J., Frenk, C. S., Lacey, C. G., Baugh, C. M., \& Cole, S. 2002, MNRAS, 333, 177

Blanton, M. R., \& Roweis, S. 2007, AJ, 133, 734

Blanton, M. R., et al. 2005, AJ, 129, 2562

Bode, P., Ostriker, J. P., \& Turok, N. 2001, ApJ, 556, 93

Bonanos, A. Z., Stanek, K. Z., Szentgyorgyi, A. H., Sasselov, D. D., \& Bakos, G. Á. 2004, AJ, 127, 861

Bullock, J. S., Kravtsov, A. V., \& Weinberg, D. H. 2000, ApJ, 539, 517

Carretta, E., \& Gratton, R. G. 1997, A\&AS, 121, 95

Chapman, S. C., Ibata, R., Lewis, G. F., Ferguson, A. M. N., Irwin, M., McConnachie, A., \& Tanvir, N. 2005, ApJ, 632, L87

Clem, J. L. 2005, Ph.D. thesis, Univ. Victoria

Coleman, M. G., et al. 2007, ApJ, 668, L43

Colín, P., Avila-Reese, V., \& Valenzuela, O. 2000, ApJ, 542, 622

Côté, P., Mateo, M., Olszewski, E. W., \& Cook, K. H. 1999, ApJ, 526, 147

Dalcanton, J. J., \& Hogan, C. J. 2001, ApJ, 561, 35

Dall'Ora, M., et al. 2003, AJ, 126, 197

Diemand, J., Kuhlen, M., \& Madau, P. 2007a, ApJ, 657, 262 2007b, ApJ, 667, 859

Faber, S. M., et al. 2003, Proc. SPIE, 4841, 1657

Fellhauer, M., et al. 2007, MNRAS, 375, 1171

Gilbert, K. M., et al. 2006, ApJ, 652, 1188

Gilmore, G., Wilkinson, M. I., Wyse, R. F. G., Kleyna, J. T., Koch, A., Evans, N. W., \& Grebel, E. K. 2007, ApJ, 663, 948

Girardi, L., Grebel, E. K., Odenkirchen, M., \& Chiosi, C. 2004, A\&A, 422, 205

Grebel, E. K., Gallagher, J. S., \& Harbeck, D. 2003, AJ, 125, 1926

Grillmair, C. J. 2006, ApJ, 645, L37

Guhathakurta, P., et al. 2006, AJ, 131, 2497

Gullieuszik, M., Held, E. V., Rizzi, L., Saviane, I., Momany, Y., \& Ortolani, S. 2007, A\&A, 467, 1025

Harris, W. E. 1996, AJ, 112, 1487

Hogan, C. J., \& Dalcanton, J. J. 2000, Phys. Rev. D, 62, 063511

Ibata, R., Chapman, S., Irwin, M., Lewis, G., \& Martin, N. 2006, MNRAS, 373, L70

Ibata, R., Martin, N. F., Irwin, M., Chapman, S., Ferguson, A. M. N., Lewis, G. F., \& McConnachie, A. W. 2007, ApJ, submitted (arXiv: 0704.1318)

Ibata, R. A., Gilmore, G., \& Irwin, M. J. 1994, Nature, 370, 194

Illingworth, G. 1976, ApJ, 204, 73

Irwin, M., \& Hatzidimitriou, D. 1995, MNRAS, 277, 1354

Irwin, M. J., et al. 2007, ApJ, 656, L13

Kamionkowski, M., \& Liddle, A. R. 2000, Phys. Rev. Lett., 84, 4525

Kauffmann, G., White, S. D. M., \& Guiderdoni, B. 1993, MNRAS, 264, 201

King, I. 1962, AJ, 67, 471

Kleyna, J., Geller, M., Kenyon, S., \& Kurtz, M. 1999, AJ, 117, 1275

Kleyna, J. T., Wilkinson, M. I., Evans, N. W., \& Gilmore, G. 2005, ApJ, 630, L141

Klypin, A., Kravtsov, A. V., Valenzuela, O., \& Prada, F. 1999, ApJ, 522, 82

Koch, A., Grebel, E. K., Wyse, R. F. G., Kleyna, J. T., Wilkinson, M. I., Harbeck, D. R., Gilmore, G. F., \& Evans, N. W. 2006, AJ, 131, 895

Koch, A., Kleyna, J. T., Wilkinson, M. I., Grebel, E. K., Gilmore, G. F., Evans, N. W., Wyse, R. F. G., \& Harbeck, D. R. 2007a, AJ, 134, 566

Koch, A., Wilkinson, M. I., Kleyna, J. T., Gilmore, G. F., Grebel, E. K., Mackey, A. D., Evans, N. W., \& Wyse, R. F. G. 2007b, ApJ, 657, 241

Koposov, S., et al. 2007, ApJ, submitted (arXiv: 0706.2687)

Kravtsov, A. V., Gnedin, O. Y., \& Klypin, A. A. 2004, ApJ, 609, 482

Lee, M. G., et al. 2003, AJ, 126, 2840

Lewis, G. F., Ibata, R. A., Chapman, S. C., McConnachie, A., Irwin, M. J., Tolstoy, E., \& Tanvir, N. R. 2007, MNRAS, 375, 1364

Łokas, E. L., Mamon, G. A., \& Prada, F. 2005, MNRAS, 363, 918

Mackey, A. D., \& Gilmore, G. F. 2003, MNRAS, 345, 747

Majewski, S. R., et al. 2007, ApJ, submitted (astro-ph/0702635)

Marcolini, A., D’Ercole, A., Brighenti, F., \& Recchi, S. 2006, MNRAS, 371, 643

Martin, N. F., Ibata, R. A., Chapman, S., Irwin, M., \& Lewis, G. F. 2007, MNRAS, 380, 281
Martin, N. F., Ibata, R. A., Irwin, M. J., Chapman, S., Lewis, G. F., Ferguson, A. M. N., Tanvir, N., \& McConnachie, A. W. 2006, MNRAS, 371, 1983

Mateo, M., Olszewski, E., Welch, D. L., Fischer, P., \& Kunkel, W. 1991, AJ, 102,914

Mateo, M., Olszewski, E. W., Pryor, C., Welch, D. L., \& Fischer, P. 1993, AJ, 105,510

Mateo, M. L. 1998, ARA\&A, 36, 435

Mayer, L., Governato, F., Colpi, M., Moore, B., Quinn, T., Wadsley, J., Stadel, J., \& Lake, G. 2001a, ApJ, 547, L123 2001b, ApJ, 559, 754

McConnachie, A. W., \& Irwin, M. J. 2006, MNRAS, 365, 1263

Mighell, K. J., \& Burke, C. J. 1999, AJ, 118, 366

Moore, B., Diemand, J., Madau, P., Zemp, M., \& Stadel, J. 2006, MNRAS, 368,563

Moore, B., Ghigna, S., Governato, F., Lake, G., Quinn, T., Stadel, J., \& Tozzi, P. 1999, ApJ, 524, L19

Muñoz, R. R., Carlin, J. L., Frinchaboy, P. M., Nidever, D. L., Majewski, S. R., \& Patterson, R. J. 2006a, ApJ, 650, L51

Muñoz, R. R., et al. 2006b, ApJ, 649, 201

Olszewski, E. W., Pryor, C., \& Armandroff, T. E. 1996, AJ, 111, 750

Page, L., et al. 2007, ApJS, 170, 335

Pryor, C., \& Meylan, G. 1993, ASP Conf. Ser. 50, Structure and Dynamics of Globular Clusters, ed. S.G. Djorgovski \& G. Meylan (San Francisco: ASP), 357

Read, J. I., Pontzen, A. P., \& Viel, M. 2006, MNRAS, 371, 885

Ricotti, M., \& Gnedin, N. Y. 2005, ApJ, 629, 259

Rutledge, G. A., Hesser, J. E., \& Stetson, P. B. 1997a, PASP, 109, 907

Rutledge, G. A., Hesser, J. E., Stetson, P. B., Mateo, M., Simard, L., Bolte, M., Friel, E. D., \& Copin, Y. 1997b, PASP, 109, 883

Ryan-Weber, E. V., Begum, A., Oosterloo, T., Pal, S. Irwin, M. J., Belokurov, V., Evans, N. W. \& Zucker, D. B. 2007, MNRAS, submitted

Sakamoto, T., \& Hasegawa, T. 2006, ApJ, 653, L29

Saviane, I., Held, E. V., \& Bertelli, G. 2000, A\&A, 355, 56

Schiavon, R. P., Barbuy, B., Rossi, S. C. F., \& Milone, A. 1997, ApJ, 479, 902

Schlegel, D. J., Finkbeiner, D. P., \& Davis, M. 1998, ApJ, 500, 525

Simon, J. D., \& Blitz, L. 2002, ApJ, 574, 726

Simon, J. D., Bolatto, A. D., Leroy, A., Blitz, L., \& Gates, E. L. 2005, ApJ, 621,757

Sohn, S. T., et al. 2007, ApJ, 663, 960

Somerville, R. S. 2002, ApJ, 572, L23

Spergel, D. N., et al. 2007, ApJS, 170, 377

Spinrad, H., \& Taylor, B. J. 1971, ApJS, 22, 445

Stoehr, F., White, S. D. M., Tormen, G., \& Springel, V. 2002, MNRAS, 335, L84

Strigari, L. E., Bullock, J. S., Kaplinghat, M., Diemand, J., Kuhlen, M., \& Madau, P. 2007a, ApJ, 669, 676

Strigari, L. E., Bullock, J. S., Kaplinghat, M., Kravtsov, A. V., Gnedin, O. Y., Abazajian, K., \& Klypin, A. A. 2006, ApJ, 652, 306

Strigari, L. E., Kaplinghat, M., \& Bullock, J. S. 2007b, Phys. Rev. D, 75, 061303

Walker, M. G., Mateo, M., Olszewski, E. W., Bernstein, R., Wang, X., \& Woodroofe, M. 2006a, AJ, 131, 2114

Walker, M. G., Mateo, M., Olszewski, E. W., Pal, J. K., Sen, B., \& Woodroofe, M. 2006b, ApJ, 642, L41

Walsh, S. M., Jerjen, H., \& Willman, B. 2007, ApJ, 662, L83

Westfall, K. B., Majewski, S. R., Ostheimer, J. C., Frinchaboy, P. M., Kunkel, W. E., Patterson, R. J., \& Link, R. 2006, AJ, 131, 375

Whiting, A. B., Hau, G. K. T., Irwin, M., \& Verdugo, M. 2007, AJ, 133, 715

Willman, B., Governato, F., Dalcanton, J. J., Reed, D., \& Quinn, T. 2004, MNRAS, 353, 639

Willman, B., et al. 2005a, ApJ, 626, L85 $2005 \mathrm{~b}, \mathrm{AJ}, 129,2692$ 2006, AJ, submitted (astro-ph/0603486)

Wu, X. 2007, ApJ, submitted (astro-ph/0702233)

York, D. G., et al. 2000, AJ, 120, 1579

Zentner, A. R., \& Bullock, J. S. 2003, ApJ, 598, 49

Zucker, D. B., et al. 2004, ApJ, 612, L121 2006a, ApJ, 643, L103 2006b, ApJ, 650, L41 2007, ApJ, 659, L21 